\title{
Resolvent analysis of stratification effects on wall-bounded shear flows
}

\author{
M. A. Ahmed $\odot,{ }^{1, *}$ H. J. Bae, ${ }^{2,1, \dagger}$ A. F. Thompson, ${ }^{3, \ddagger}$ and B. J. McKeon ${ }^{1, \S}$ \\ ${ }^{1}$ Graduate Aerospace Laboratories, California Institute of Technology, Pasadena, California 91125, USA \\ ${ }^{2}$ Institute of Applied Computational Science, Harvard University, Cambridge, Massachusetts 02138, USA \\ ${ }^{3}$ Geophysical and Planetary Sciences, California Institute of Technology, Pasadena, California 91125, USA
}

(Received 15 January 2021; accepted 12 July 2021; published 11 August 2021)

\begin{abstract}
The interaction between shear-driven turbulence and stratification is a key process in a wide array of geophysical flows with spatiotemporal scales that span many orders of magnitude. A quick numerical model prediction based on external parameters of stratified boundary layers could greatly benefit the understanding of the interaction between velocity and scalar flux at varying scales. For these reasons, here we use the resolvent framework [McKeon and Sharma, J. Fluid Mech., 658 (2010)] to investigate the effects of an active scalar on incompressible wall-bounded turbulence. We obtain the state of the flow system by applying the linear resolvent operator to the nonlinear terms in the governing Navier-Stokes equations with the Boussinesq approximation. This extends the formulation to include the scalar advection equation with the scalar component acting in the wall-normal direction in the momentum equations [Dawson, Saxton-Fox and McKeon, AIAA Fluid Dyn. Conf. 4042 (2018)]. We use the mean velocity profiles from a direct numerical simulation (DNS) of a stably stratified turbulent channel flow at varying friction Richardson number $\mathrm{Ri}_{\tau}$. The results obtained from the resolvent analysis are compared to the premultiplied energy spectra, autocorrelation coefficient, and the energy budget terms obtained from the DNS. It is shown that despite using only a very limited range of representative scales, the resolvent model is able to reproduce the balance of energy budget terms as well as provide meaningful insight into coherent structures occurring in the flow. Computation of the leading resolvent models, despite considering a limited range of scales, reproduces the balance of energy budget terms, provides meaningful predictions of coherent structures in the flow, and is more cost-effective than performing full-scale simulations. This quick model can provide a further understanding of stratified flows with only information about the mean profile and prior knowledge of energetic scales of motion in the neutrally buoyant boundary layers.
\end{abstract}

DOI: 10.1103/PhysRevFluids.6.084804

\section{INTRODUCTION}

Stratification plays an important role in both atmospheric and oceanic flows, and in particular, near boundaries, it has a significant effect on turbulence production, propagation, and decay. The interaction between shear-driven turbulence and stratification is a key process in a wide array of relevant geophysical flows for which the spatiotemporal scales span many orders of magnitude. In the atmosphere, stable boundary layers can be generated by the advection of warm air over a colder

\footnotetext{
*arslan@ caltech.edu

†jbae@caltech.edu

†andrewt@caltech.edu

§mckeon@caltech.edu
} 
surface. Stably stratified atmospheric boundary layers are observed during clear nights as a result of radiative cooling of the ground surface $[1,2]$. Oceans, unlike the lower atmosphere, are heated from above and are stably stratified outside of small regions of localized convection $[3,4]$. This stable stratification shapes many aspects of ocean turbulence although sampling is considerably sparser than the atmosphere [5].

Classical understanding of stably stratified boundary layers is well described in a number of textbooks [6-9] and reviews [10,11]. However, fundamental features of the stably stratified turbulent boundary layer still remain elusive from a modeling standpoint. The strong intermittency observed in stable boundary layers causes the upper portion of the boundary layer to decouple from the near-wall region due to the inhibition in vertical mixing $[8,12,13]$. Strong stable stratification also significantly changes the flow structures prevalent in a boundary layer with additional features becoming prominent such as large-scale intermittency, gravity waves and Kelvin-Helmholtz instabilities [12], and the near parallel downstream tilting of flow structures [14-16]. At the interface between the boundary layer and an outer stratified region, a pycnocline can develop with the stratification principally acting to limit the boundary layer height [17].

One way to study the stably stratified turbulent boundary layer is through on-site experiments. Researchers in the past decades have conducted field experiments in the stably stratified atmospheric boundary layer to study turbulent energy budgets [18], heat and momentum transfer [19], regime characterization [12,20], flow structures [14], and the complexities of atmospheric stable boundary layers [21]. Measurements of turbulence quantities in the ocean near the bottom boundary are difficult to measure, and as such the literature is sparse. Smedman et al. [22], using data from a marine coastal experiment over the Baltic sea, found that the near-wall turbulence was virtually independent of forcing from large-scale structures embedded in the flow. Experiments performed in the northern bay of San Francisco [23] found that active turbulence is confined near the wall. Bluteau et al. [24] conducted a 21-day field study of the stratified flow dynamics near the bottom boundary on the continental slope of the Australian North West Shelf; the authors found that measurements closest to the seabed were described by the log law of the wall, and that stratification had very little influence on turbulent shear production near the seabed. Furthermore, their results showed that the production of turbulence weakened further from the seabed as the local buoyancy frequency increased. Additionally, tidal channel experiments [25] demonstrated that the production of turbulent kinetic energy is generally greatest near the bottom boundary while the buoyancy flux is weakest in this region. Still, real-world atmospheric and oceanic boundary layers are complicated by nonturbulent motions occurring simultaneously on a variety of scales, such as wave motions [26] or tidal bores [27], the possible importance of radiative flux divergence of the air within the boundary layer, surface condensation, and variable cloudiness $[10,11,28]$. In order to isolate instances where the secondary effects are minimized, restrictions on nonstationarity or conditions on the minimum allowed value of turbulence energy may be applied to the data collected. Nonetheless, certain assumptions that are applied for analyses of these real-world stratified boundary layers are not always valid. As such, researchers supplement their work with laboratory experiments as well as simulations.

Laboratory experiments of stratified wall-bounded flows show that buoyancy effects play an important role in the transfer of heat and momentum in both the inner and outer layers of the boundary layer [29-33]. In general, the experiments show that with increasing stratification, the turbulence shear production rate is strongly affected by buoyancy and greatly reduced far from the wall. One measure of stratification strength is the local gradient Richardson number, $\mathrm{Ri}_{g}$. Since shear originates at the wall, the local gradient Richardson number, which is inversely proportional to the shear, is generally smaller in the near-wall region as the shear term overpowers the buoyancy term. The stabilizing effect of stratification has a greater impact farther from the wall. Indeed, works listed here demonstrated that velocity fluctuations become weaker further from the wall, and in some cases, turbulence intensity is reduced as the buoyancy frequency in the system is increased. 
Additionally, the experiments of Komori et al. [32] show that the correlation coefficients associated with the Reynolds shear stress approach zero at values of $\mathrm{Ri}_{g} \simeq 0.2-0.3$.

There have been many large-eddy simulations (LES) [34-39] and direct numerical simulations (DNS) [40-44] of density stratified channel flows. The results support the experimental observations: strengthening the stratification leads to the reduction (or even suppression) of turbulent velocity fluctuations further from the wall. Garg et al. [34] showed in their work that the mean velocity profiles of the stratified channel were similar in the near-wall region but differed in the logarithmic region. The difference is characterized by a reduction in the value of both the slope of the log-law of the mean velocity and the gradient of the mean velocity profile. It should be noted that the authors used the friction Richardson number to categorize the stratification strengths investigated in their simulations and concluded that the friction Richardson number is superior to the local gradient Richardson number in characterizing flow regimes as it is a global flow property. There have also been DNS studies of homogeneous stably stratified flow which exhibit similar results [45-47]. Holt et al. [47] found that as the gradient Richardson number increases, the production and turbulent kinetic energy decrease. Their results also demonstrated that the production of streamwise velocity fluctuations at low gradient Richardson numbers is due to the down-gradient Reynolds stress. Furthermore, the kinetic energy is redistributed to the spanwise and vertical components via pressure-strain interactions.

Previous research has also shown that stable stratification inhibits the self-sustained processes that exist in turbulent channel flows, studied by, e.g., [48,49]. The coherent motions that persist during the self-sustaining processes exist as quasistreamwise roll vortices in the sublayer, and they take the form of hairpin vortices and double roller eddies above the sublayer. Bakas et al. [50] found that the energy of the roll structures is suppressed by stable stratification compared to neutral stratification. In addition, Eaves and Caulfield [51] demonstrated that stable stratification disrupts the self-sustaining processes through an inhibition of vertical motions.

Performing experiments (both on-site and in laboratories) of stratified wall-bounded turbulence can be challenging for reasons such as topography or secondary effects and simulations suffer from computational constraints. Moreover, laboratory experiments and simulations can attain only a limited range of Reynolds and Richardson numbers that are often orders of magnitude smaller than real-world geophysical phenomena. A quick numerical model prediction of key features of stratified boundary layers could greatly benefit the understanding of the interaction between velocity and scalar flux at varying scales. Recently, works targeted towards reconstructing velocity fields from a few spatial probe measurements and resolvent modes have been successful [52-54]. While these studies have been focused on incompressible neutrally buoyant cases, we expect the methodology to extend to stratified flows as well. For these reasons, in this paper, we aim to explore the interaction between velocity and scalar fluctuations using the resolvent model [55].

The resolvent model provides an optimal basis, in an energy sense, that allows an in-depth comparison of the underlying mechanisms in the flow. Moreover, the model is computationally efficient where only the singular vectors corresponding to the largest singular value are required to obtain the leading order model. Resolvent analysis has been widely applied to a range of flow configurations to identify dominant flow structures and the underlying forcing, e.g., Refs. [55-61], and has been reviewed in detail in Refs. [62,63]. The resolvent model does not need $a$ priori knowledge of the flow fluctuations and the assumption of white-noise forcing is often employed. Recently, Morra et al. [64] performed DNS of unstratified turbulent channel flows at $\operatorname{Re}_{\tau}=179$ and used the cross-spectral density to quantify the nonlinear forcing term. They showed that the nonlinear forcing is structured and the resulting resolvent response modes are in very good agreement with DNS data. Similarly, Nogueira et al. [61] also showed that the forcing is structured in turbulent plane Couette flow.

We use the model to provide analysis of the flow using only mean quantities, which are easy to obtain even in field experiments, along with knowledge from the energetics of the unstratified case, which is better documented than the stably stratified case. The predictions from the resolvent model are then compared to the flow statistics from a DNS of a stably stratified turbulent channel flow. Note 
that we do not compute the nonlinear forcing term from DNS to use as input in the resolvent model. The Reynolds number under consideration in the current study is considerably lower than those observed in geophysical flows, which is dictated by the available DNS data for comparison, rather than by the resolvent model. Resolvent analysis of unstratified wall-bounded flows shows that the results of the model are still relevant for moderate Reynolds numbers [65] with the resolvent modes in the logarithmic layer showing self-similar behavior. We expect the capability of the model in stably stratified regimes to extend to higher Reynolds numbers as well.

The paper is organized as follows. In Sec. II we introduce the resolvent framework with the inclusion of the scalar advection-diffusion equation and discuss the relevant energy norm, boundary conditions, and computational methods. In Sec. III A we examine the sensitivity of the low-rank properties of the resolvent operator to the stable stratification strength and compare these properties with the most energetic scales in each flow. In Sec. III B we analyze the characteristics of the forcing and response modes of both velocity and scalar. We compare the mode shapes with correlations obtained from DNS data. In Sec. III C we study the turbulent kinetic energy budget in the resolvent formulation and compare the results with the energy budget obtained from the DNS data. Finally, our conclusions on the application of the resolvent framework to a stably stratified boundary layer are given in Sec. IV.

\section{MODELING ACTIVE SCALAR DYNAMICS IN THE NAVIER-STOKES EQUATIONS}

\section{A. Navier-Stokes equation with active scalar}

We consider a density-stratified turbulent channel flow where the density acts in the direction of gravitational acceleration. We use a Cartesian coordinate system $\boldsymbol{x}=(x, y, z)$ such that the force of gravity acts in the $-y$ direction, with $x, y$, and $z$ being the streamwise, wall-normal, and spanwise directions, respectively. The governing equations are given by the nondimensional Navier-Stokes equation under the Boussinesq approximation,

$$
\begin{aligned}
\frac{\partial \tilde{\boldsymbol{u}}}{\partial t}+(\tilde{\boldsymbol{u}} \cdot \nabla) \tilde{\boldsymbol{u}} & =-\nabla \tilde{p}+\frac{\nabla^{2} \widetilde{\boldsymbol{u}}}{\operatorname{Re}_{\tau}}-\operatorname{Ri}_{\tau} \tilde{\rho} \boldsymbol{e}_{y}, \\
\frac{\partial \widetilde{\rho}}{\partial t}+(\tilde{\boldsymbol{u}} \cdot \nabla) \tilde{\rho} & =\frac{\nabla^{2} \widetilde{\rho}}{\operatorname{Re}_{\tau} \operatorname{Pr}}, \\
\nabla \cdot \widetilde{\boldsymbol{u}} & =0 .
\end{aligned}
$$

Here $\tilde{\boldsymbol{u}}=(\tilde{u}, \tilde{v}, \tilde{w})$ is the instantaneous velocity vector in the reference system $(x, y, z), t$ is time, $\tilde{p}$ is the kinematic pressure field that remains after removing the part that is in hydrostatic balance with the mean density field, $\tilde{\rho}$ is the density deviation from the reference density $\rho_{0}\left(\widetilde{\rho} \ll \rho_{0}\right)$, and $\boldsymbol{e}_{y}$ is the unit vector acting in the $y$-direction. The velocity and length scales are nondimensionalized using the friction velocity $u_{\tau}$ and channel half-height $\delta$, respectively, and the density is nondimensionalized using $\Delta \rho$, the difference in density between the two channel walls. We define the walls to be located at $y=0$ and $y=2$. The nondimensional quantities are given by the Reynolds, Prandtl, and Richardson numbers, defined as

$$
\operatorname{Re}_{\tau}=\frac{u_{\tau} \delta}{\nu}, \quad \operatorname{Pr}=\frac{v}{\gamma}, \quad \operatorname{Ri}_{\tau}=\frac{g \Delta \rho \delta}{\rho_{0} u_{\tau}^{2}},
$$

where $v$ is the kinematic viscosity, $\gamma$ is the molecular diffusivity of density, and $g$ is the acceleration due to gravity.

\section{B. Resolvent framework with an active scalar}

The total fields $\tilde{\boldsymbol{u}}, \tilde{p}$, and $\widetilde{\rho}$ can be split into mean and fluctuating parts as

$$
\tilde{\boldsymbol{u}}(\boldsymbol{x}, t)=\overline{\boldsymbol{u}}(y)+\boldsymbol{u}(\boldsymbol{x}, t)
$$




$$
\begin{aligned}
& \widetilde{p}(\boldsymbol{x}, t)=\bar{p}(y)+p(\boldsymbol{x}, t), \\
& \widetilde{\rho}(\boldsymbol{x}, t)=\bar{\rho}(y)+\rho(\boldsymbol{x}, t),
\end{aligned}
$$

where the mean is taken in the homogeneous directions, $x$ and $z$, and time. Note that $\overline{\boldsymbol{u}}=(\bar{u}, \bar{v}, \bar{w})$ and $\bar{v}=\bar{w}=0$. We substitute the decomposed variables into Eq. (1) to obtain the fluctuation equations

$$
\begin{aligned}
\partial_{t} \boldsymbol{u}+(\bar{u} \cdot \nabla) \boldsymbol{u}+(\boldsymbol{u} \cdot \nabla) \bar{u} & =-\nabla p+\frac{\nabla^{2} \boldsymbol{u}}{\operatorname{Re}_{\tau}}-\operatorname{Ri}_{\tau} \rho \boldsymbol{e}_{y}+\boldsymbol{f}_{\boldsymbol{u}}, \\
\partial_{t} \rho+(\bar{u} \cdot \nabla) \rho+(\boldsymbol{u} \cdot \nabla) \bar{\rho} & =\frac{\nabla^{2} \rho}{\operatorname{Re}_{\tau} \operatorname{Pr}}+f_{\rho}, \\
\nabla \cdot \boldsymbol{u} & =0,
\end{aligned}
$$

where $\boldsymbol{f}_{\boldsymbol{u}}=-\boldsymbol{u} \cdot \nabla \boldsymbol{u}$ and $f_{\rho}=-\boldsymbol{u} \cdot \nabla \rho$ are the nonlinear terms.

Taking the Fourier transform of the fluctuation equations above in homogeneous directions and time, the variables can be expressed as

$$
\left[\begin{array}{l}
\boldsymbol{u}(x, y, z, t) \\
p(x, y, z, t) \\
\rho(x, y, z, t)
\end{array}\right]=\iiint_{-\infty}^{\infty}\left[\begin{array}{l}
\hat{\boldsymbol{u}}\left(y ; k_{x}, k_{z}, \omega\right) \\
\hat{p}\left(y ; k_{x}, k_{z}, \omega\right) \\
\hat{\rho}\left(y ; k_{x}, k_{z}, \omega\right)
\end{array}\right] e^{\mathrm{i}\left(k_{x} x+k_{z} z-\omega t\right)} d k_{x} d k_{z} d \omega,
$$

for $\boldsymbol{k}=\left(k_{x}, k_{z}, \omega\right) \neq(0,0,0)$, where $(\hat{\bullet})$ denotes the Fourier transformed variables. Here the streamwise and spanwise wavenumbers are $k_{x}$ and $k_{z}$, respectively, and $\omega$ is the temporal frequency defined as $\omega=c k_{x}$, where $c$ is the wave speed. The streamwise and spanwise wavelengths are defined as $\lambda_{x}=2 \pi / k_{x}$ and $\lambda_{z}=2 \pi / k_{z}$, respectively. Critical layers can be identified when the wave speed $c$ is equivalent to the mean velocity, i.e., $y_{c}$ is the critical layer location for wave speed $c=\bar{u}\left(y_{c}\right)$. Assuming the mean velocity and density profiles are known, the fluctuations equations are expressed compactly in a linear equation as

$$
-\mathrm{i} \omega \hat{\boldsymbol{q}}-\mathcal{A} \hat{\boldsymbol{q}}=\hat{\boldsymbol{f}}
$$

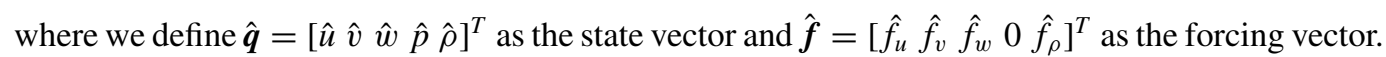
The linear operator is given by

$$
\mathcal{A}=\left(\begin{array}{ccccc}
A & -\partial \bar{u} / \partial y & 0 & -\mathrm{i} k_{x} & 0 \\
0 & A & 0 & -D_{y} & -\mathrm{Ri}_{\tau} \\
0 & 0 & A & -\mathrm{i} k_{z} & 0 \\
-\mathrm{i} k_{x} & -D_{y} & -\mathrm{i} k_{z} & 0 & 0 \\
0 & -\partial \bar{\rho} / \partial y & 0 & 0 & A_{\rho}
\end{array}\right),
$$

where

$$
\begin{aligned}
A & =-\mathrm{i} k_{x} \bar{u}+\frac{\hat{\Delta}}{\operatorname{Re}_{\tau}}, \\
A_{\rho} & =-\mathrm{i} k_{x} \bar{u}+\frac{\hat{\Delta}}{\operatorname{Re}_{\tau} \operatorname{Pr}},
\end{aligned}
$$

$D_{y}$ is the wall-normal derivative operator and $\hat{\Delta} \equiv D_{y y}-k_{\perp}^{2}$ is the Laplacian with $k_{\perp}^{2}=k_{x}^{2}+k_{z}^{2}$. The block matrix $\mathcal{A}$ describes the linear dynamics of the system. Equation (6) can be rearranged to yield

$$
\hat{\boldsymbol{q}}=\mathcal{H}(\boldsymbol{k}) \hat{\boldsymbol{f}},
$$

where $\mathcal{H}(\boldsymbol{k})=(-\mathrm{i} \omega I-\mathcal{A})^{-1}$ is the resolvent of the linear operator and $I$ is the identity matrix. A related analysis has been performed in Ref. [66]. 
From Eq. (9) we wish to find a decomposition of the resolvent operator that enables us to identify high-gain input and output modes with respect to the linear operator. For resolvent analysis, this is given by the Schmidt decomposition. However, this decomposition must be accompanied by a choice of inner product and the corresponding norm. The natural and physically meaningful norm is given by the nondimensionalized energy norm, which is the sum of kinetic and potential energies $[67,68]$

$$
\frac{1}{2}\|\boldsymbol{q}\|_{E}^{2}=\frac{1}{2}(\boldsymbol{q}, \boldsymbol{q})_{E}=\frac{1}{2} \int_{0}^{2}\left[u^{*} u+v^{*} v+w^{*} w+\operatorname{Ri}_{\tau}\left(\rho^{*} \rho\right)\right] d y,
$$

where $(\cdot)^{*}$ denotes the conjugate transpose.

We perform the Schmidt decomposition of the resolvent operator $\mathcal{H}$ to generate a basis based on the most highly amplified forcing and response directions such that

$$
\mathcal{H}(\boldsymbol{k})=\sum_{j=1}^{\infty} \sigma_{j}(\boldsymbol{k}) \hat{\boldsymbol{\psi}}_{j}(y ; \boldsymbol{k}) \hat{\boldsymbol{\phi}}_{j}^{*}(y ; \boldsymbol{k})
$$

where the right and left Schmidt bases (or singular vectors in the discrete case) are given by $\hat{\boldsymbol{\phi}}_{j}$ and $\hat{\boldsymbol{\psi}}_{j}$ along with their corresponding gains $\sigma_{j}$. The singular values are in descending order such that $\sigma_{1} \geqslant \sigma_{2} \geqslant \cdots \geqslant 0$. The forcing and resolvent modes are orthonormal such that

$$
\left(\hat{\boldsymbol{\phi}}_{j}, \hat{\boldsymbol{\phi}}_{k}\right)_{E}=\left(\hat{\boldsymbol{\psi}}_{j}, \hat{\boldsymbol{\psi}}_{k}\right)_{E}=\delta_{j k},
$$

where $\delta_{j k}$ denotes the Kronecker delta. The basis pair defined above is used to decompose the nonlinear forcing and response field at a specified wavenumber triplet as

$$
\begin{aligned}
& \hat{\boldsymbol{f}}(y ; \boldsymbol{k})=\sum_{j=1}^{\infty} \hat{\boldsymbol{\phi}}_{j}(y ; \boldsymbol{k}) \chi_{j}(\boldsymbol{k}), \\
& \hat{\boldsymbol{q}}(y ; \boldsymbol{k})=\sum_{j=1}^{\infty} \chi_{j}(\boldsymbol{k}) \sigma_{j}(\boldsymbol{k}) \hat{\boldsymbol{\psi}}_{j}(y ; \boldsymbol{k}) .
\end{aligned}
$$

Here $\chi_{j}$ is a projection variable that is obtained by projecting the nonlinear forcing onto the forcing modes and subsequently use to weight the response modes. Note that the largest energy is obtained when the forcing is aligned with the leading singular vector, i.e., when $\chi_{j}=\delta_{j 1}$. While the values of $\chi_{j}$ may be obtained from time-resolved flow fields, for simplicity, we assume white-noise forcing in the wall-parallel directions and time throughout the paper.

\section{Computational approach}

\section{Mean velocity and density profiles}

Mean velocity and density profiles are required to close the resolvent model. We obtain the one-dimensional mean velocity and density profiles from a DNS of a stratified turbulent channel at $\operatorname{Re}_{\tau}=180$ for a wide range of $\mathrm{Ri}_{\tau}$. The simulations are performed by discretizing the incompressible Navier-Stokes equations with a staggered, second-order accurate, central finite-difference method in space [69], and an explicit third-order accurate Runge-Kutta method for time advancement [70]. The system of equations is solved via an operator splitting approach [71]. The code has been verified for neutrally buoyant cases in Refs. [72,73].

Periodic boundary conditions are imposed in the streamwise and spanwise directions, the no-slip and no-penetration condition with $\tilde{\rho}=0$ is applied at the bottom boundary, and a no-slip and no-penetration condition with $\tilde{\rho}=1$ is applied at the top boundary. The streamwise, wall-normal, and spanwise domain sizes are $L_{x}=4 \pi, L_{y}=2$, and $L_{z}=2 \pi$ respectively. The grid spacings in the streamwise and spanwise directions are uniform with $\Delta x^{+}=8.8$ and $\Delta z^{+}=4.4$; nonuniform 
TABLE I. Comparison of our DNS and the results of Ref. [44] denoted under columns titled GV11, both at $\operatorname{Re}_{\tau}=180 . \operatorname{Re}_{B}$ is the bulk Reynolds number defined as $u_{B} \delta / v$ where the bulk velocity is $u_{B}=\int_{0}^{2} \bar{u} d y / 2 . \mathrm{Ri}_{B}$ is the bulk Richardson number, which is defined as $\mathrm{Ri}_{B}=g \Delta \rho \delta /\left(2 \rho_{0} u_{B}^{2}\right)$. Nu is the Nusselt number defined as $\mathrm{Nu}=2 \delta q_{w} /(\gamma \Delta \rho)$, where $q_{w}$ is the density flux at the wall. For laminar flow $\mathrm{Nu}=1$.

\begin{tabular}{|c|c|c|c|c|c|c|}
\hline \multirow[b]{2}{*}{$\mathrm{Ri}_{\tau}$} & \multicolumn{2}{|c|}{$\mathrm{Re}_{B}$} & \multicolumn{2}{|c|}{$\mathrm{Ri}_{B}$} & \multicolumn{2}{|c|}{$\mathrm{Nu}$} \\
\hline & GV11 & DNS & GV11 & DNS & GV11 & DNS \\
\hline 0 & 2820 & 2823 & 0.000 & 0.000 & 6.03 & 6.08 \\
\hline 10 & - & 2970 & - & 0.018 & - & 4.78 \\
\hline 18 & 3043 & 3060 & 0.031 & 0.031 & 4.02 & 4.15 \\
\hline 60 & 3436 & 3473 & 0.082 & 0.081 & 2.80 & 2.82 \\
\hline 100 & - & 3850 & - & 0.109 & - & 2.37 \\
\hline
\end{tabular}

meshes are used in the wall-normal direction, with the grid stretched toward the wall according to a hyperbolic tangent distribution with $\min \left(\Delta y^{+}\right)=0.31$ and $\max \left(\Delta y^{+}\right)=5.19$, where the superscript + indicates length scales in wall units normalized by $v / u_{\tau}$ rather than $\delta$. A constant pressure gradient is applied to drive the flow. The simulation was run over 100 eddy-turnover times, defined as $\delta / u_{\tau}$, after transients. The mean quantities computed from the simulations are statistically stationary and converged.

The work of García-Villalba and del Álamo [44] at $\operatorname{Re}_{\tau}=180$ is used to validate the results. The comparison of a few key quantities is shown in Table I, which indicates a good agreement for all Richardson numbers. The mean and root-mean-squared streamwise velocity and density profiles are shown in Fig. 1 for all current cases and select cases from Ref. [44] and show good agreement among all statistics. The mean profiles are linearly stable in each case.

\section{Resolvent mode computation}

The Schmidt decomposition of the resolvent operator outlined in Sec. II B is numerically implemented as the singular value decomposition (SVD) of the matrix $(-i \omega I-\mathcal{A})^{-1}$, where each block of the linear operator $\mathcal{A}$ in Eq. (7) is a $N_{y} \times N_{y}$ matrix with $N_{y}$ being the number of points in the wall-normal direction. The discrete system limits the number of singular values to $5 N_{y}$ because the state vector $\hat{\boldsymbol{q}} \in \mathbb{C}^{5 N_{y} \times 1}$. The wall-normal points are chosen as Chebyshev nodes. The nondimensional-energy norm is similarly discretized using a numerical quadrature. See Trefethen and Embree [74] for more details on the numerical computation of the resolvent modes. In this study, after conducting a grid convergence study examining the singular values, we selected a wall-normal grid resolution of $N_{y}=400$. Thus, the computational cost of the resolvent mode computation is at most $O\left(N_{y}^{3}\right)$ (less if randomized algorithms are employed [65,75]), often requiring only a leading order singular value decomposition (see Sec. III A for more information) and can be performed in seconds on a personal computer.

The discretized linear operator is constructed using Chebyshev differentiation matrices and is shifted to integrate between $y \in[0,2]$ rather than $y \in[-1,1]$. The mean velocity and density profiles obtained from DNS as well as their wall-normal derivatives are interpolated to the Chebyshev grid points to form the resolvent operator as in Eq. (7). The no-slip and no-penetration boundary conditions for the fluctuating velocities and density, i.e., $u, v, w, \rho=0$, are applied at the walls.

In the case of a turbulent channel, due to the symmetry in the geometry, the resolvent modes appear in pairs that can be linearly combined to produce symmetric and antisymmetric modes. Depending on the support of these modes, the singular values may be identical or similar in magnitude. For the results in the following sections, only results in the bottom half-channel will be shown, but the corresponding upper half-channel results are analogous in all cases.

The results from the analysis of resolvent modes are compared to DNS data. In particular we compare the energy contained in the leading resolvent modes to the the energy spectra, the mode 


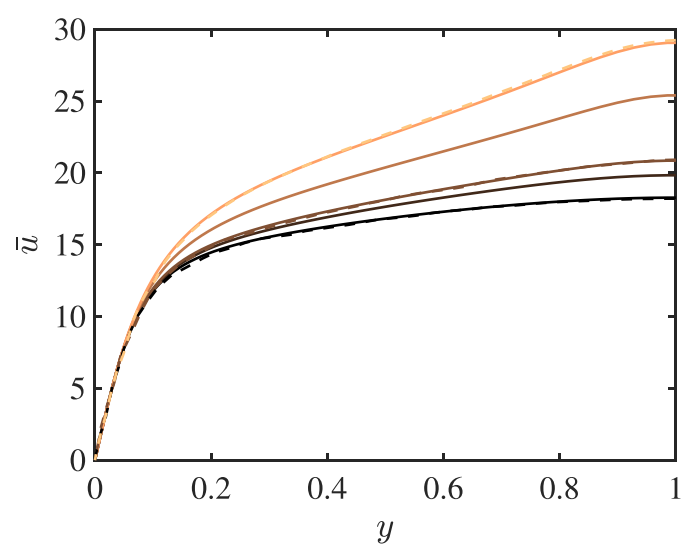

(a)

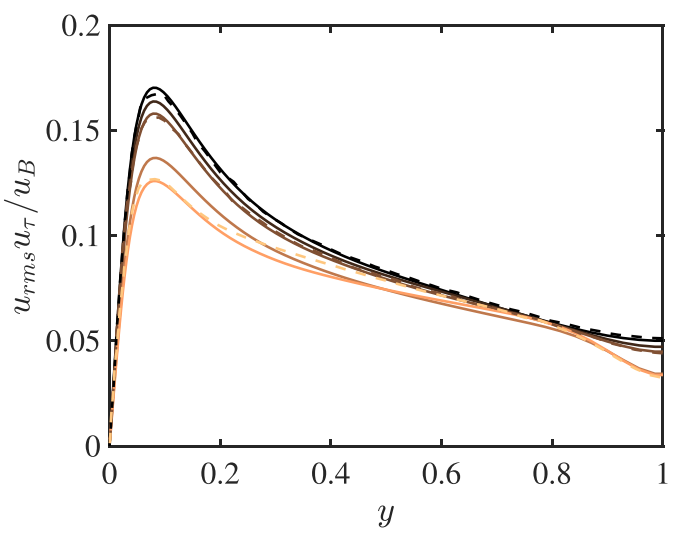

(c)

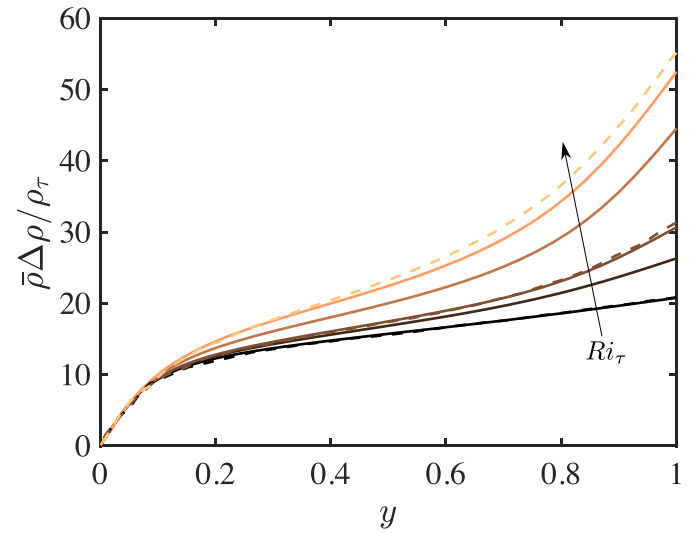

(b)

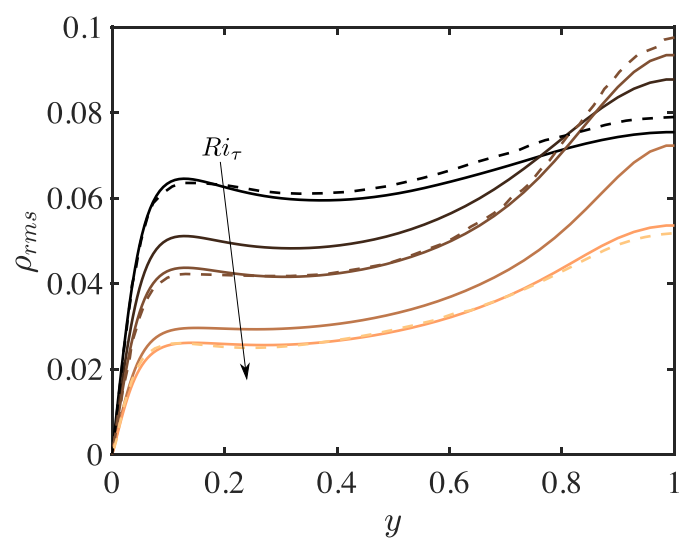

(d)

FIG. 1. Mean (a) streamwise velocity and (b) density profiles and root-mean-square (r.m.s.) (c) streamwise velocity and (d) density profiles from the current DNS for $\mathrm{Ri}_{\tau}=0,10,18,60,100$ (solid lines darker to lighter), compared to the mean profiles of Ref. [44] for $\mathrm{Ri}_{\tau}=0,18,120$ (dashed lines darker to lighter). The friction density is defined as $\rho_{\tau}=q_{w} / u_{\tau}$, where $q_{w}$ is the density flux at the wall.

shapes to the proper orthogonal decomposition (POD) modes and correlation coefficients, and energy budgets from resolvent modes to those computed from DNS data.

\section{RESULTS}

In this section, we explore how the resolvent analysis provides insight into changes in flow characteristics with increasing stratification from only a limited range of representative scales. We compare (1) the resolvent energy spectra, obtained from the ratio of the energy in the leading resolvent response mode pair to the total response, $\left(\sigma_{1}^{2}+\sigma_{2}^{2}\right) / \sum_{j} \sigma_{j}^{2}$, to the premultiplied energy spectra of the DNS, (2) the structure identified by the leading resolvent mode to the correlation computed from DNS, and (3) the energy budgets of the resolvent modes to that of the DNS.

In order for full representation of the system, a wide range of scales, as well as information of all other subsequent modes in addition to the leading resolvent modes, are necessary [55,62]. However, the goal here is to provide a quick model for characterizing the flow. The simplest and quickest 
model can be provided via a rank-one approximation, where only the leading resolvent mode is computed. Thus, our focus will be on the representation given by the leading resolvent mode for a limited number of scales.

\section{A. Resolvent energy spectra}

The resolvent norm, $\sigma_{1}$, quantifies the system's sensitivity to temporal forcing. Here we use $\sqrt{\sigma_{1}^{2}+\sigma_{2}^{2}}$ (to account for pairs of singular vectors from geometric symmetry), of the resolvent operator $\mathcal{H}$ to study the energetic response from broadband forcing associated with the first two modes. The resolvent operator $\mathcal{H}$ can be described as low-rank if the majority of its response to white-noise forcing in the wall-normal direction is captured by the first few response modes. Theoretically, there are an infinite number of singular values and corresponding modes because the wall-normal coordinate is continuous. However, not all of the singular vectors are energetically significant. As described in Sec. II B, a self-sustaining representation of the flow will correspond to a weighted assembly of forcing modes rather than a white-noise forcing [61]; however, past studies have shown that broadband forcing is successful in identifying the important component of the flow, e.g., Ref. [55,59]. McKeon and Sharma [55] demonstrated that the characteristics of the leading response modes for a range of wavenumber-frequency combinations agree with experimental observations in pipe flow and with scaling concepts in wall-bounded turbulence. Moarref et al. [65] showed that the first two resolvent modes account for more than $80 \%$ of the total response in a channel. Bae et al. [59] investigated the low-rank nature of a compressible turbulent boundary layer and highlighted the similarities in the region where the low-rank approximation is valid for the incompressible regime.

Assuming the resolvent operator is low-rank $\left(\sigma_{1} \simeq \sigma_{2} \gg \sigma_{3}\right)$ allows us to approximate the operator as

$$
\mathcal{H}(\boldsymbol{k}) \approx \sigma_{1} \hat{\boldsymbol{\psi}}_{1} \hat{\boldsymbol{\phi}}_{1}^{*}+\sigma_{2} \hat{\boldsymbol{\psi}}_{2} \hat{\boldsymbol{\phi}}_{2}^{*}
$$

for each $\boldsymbol{k}$ since most of the energy in the system is modeled by the principal singular value. The low-rank behavior of $\mathcal{H}$ is typically representative of there being a dynamically significant physical, spatiotemporal structure at the scale dictated by $\boldsymbol{k}$.

To study the variation in the low-rank behavior for different magnitudes of stratification, we plot the energetic contribution of the principal response mode to the total response in the model for a given $\boldsymbol{k}$ quantified by $\left(\sigma_{1}^{2}+\sigma_{2}^{2}\right) / \Sigma_{j} \sigma_{j}^{2}$ for a range of wall-parallel wavelengths (Fig. 2). The leading response mode pair accounts for more than $80 \%$ of the total response over a large range of homogeneous wavelengths for the three wave speeds selected.

The range of wavenumbers for which the resolvent operator is low-rank changes significantly with stratification. In the neutrally buoyant case $\left(\mathrm{Ri}_{\tau}=0\right)$, we see that $\mathcal{H}$ is low-rank in a range of moderate-to-large streamwise wavelengths. For the neutrally buoyant case, it is known that the low-rank region coincides with the most energetic wavenumbers from the premultiplied energy spectra of a turbulent channel [65]. As the friction Richardson number first increases, the low-rank behavior shifts to only a small range of streamwise wavelengths. We see a similar phenomenon in the premultiplied streamwise energy spectra from the DNS (Fig. 3), where with increasing $\mathrm{Ri}_{\tau}$, the larger streamwise wavelength content is suppressed. This was also observed in the premultiplied energy spectra of Ref. [44] for a wider range of $\operatorname{Re}_{\tau}$ and $\mathrm{Ri}_{\tau}$.

However, after $\mathrm{Ri}_{\tau}=18$, the low-rank behavior of the principal resolvent modes intensifies along a vertical band $\lambda_{x} / \delta \geqslant 1$ until the system becomes low-rank at large spanwise wavelengths with almost no low-rank behavior below the green dashed line in Fig. $2\left(\lambda_{x}=15 \lambda_{z}, 10 \lambda_{z}\right.$, and $5 \lambda_{z}$ for $y^{+}=15,30$, and 100, respectively). This seems to indicate a low-rank behavior in structures that are descriptive of quasi-two-dimensional flow where $\lambda_{z} \gg \lambda_{x}$. Hopfinger [77] details the emergence 

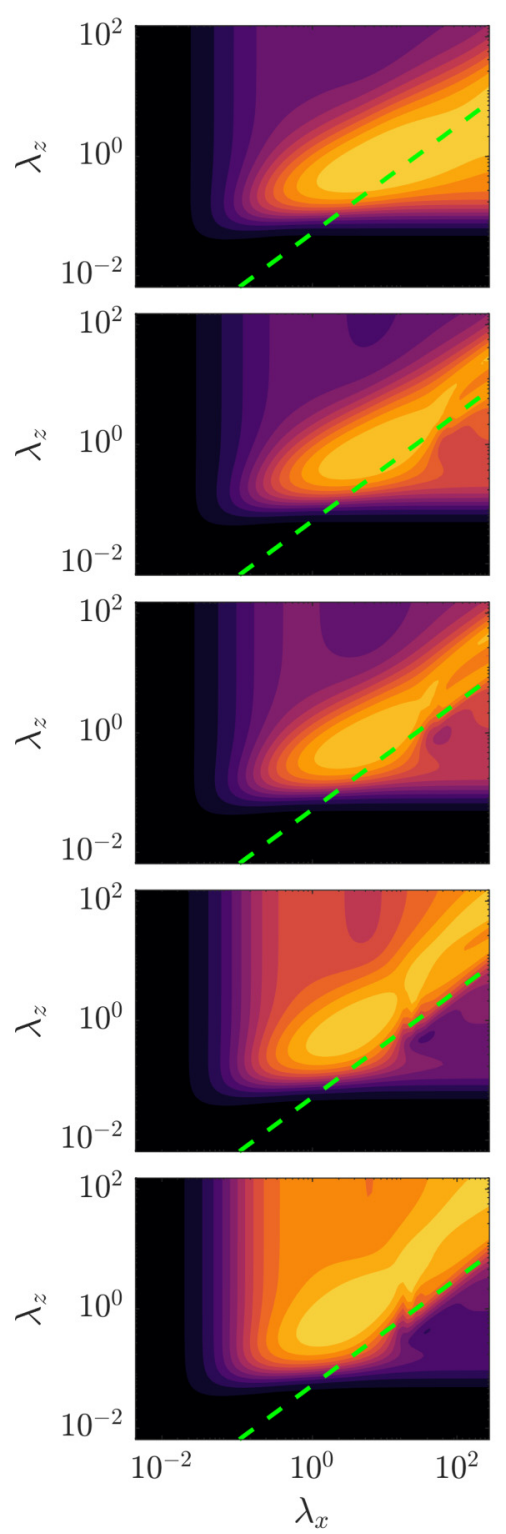

(a)
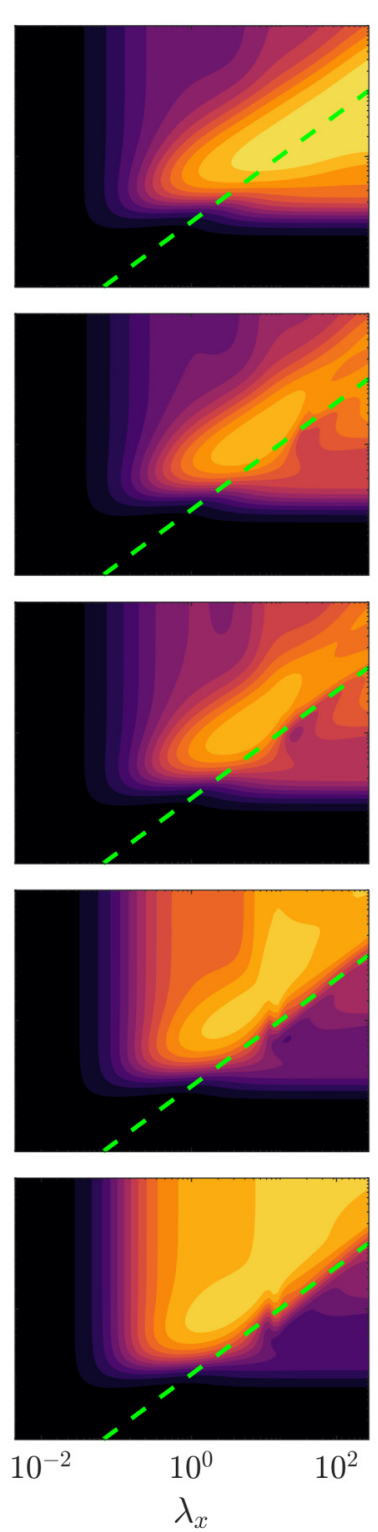

(b)
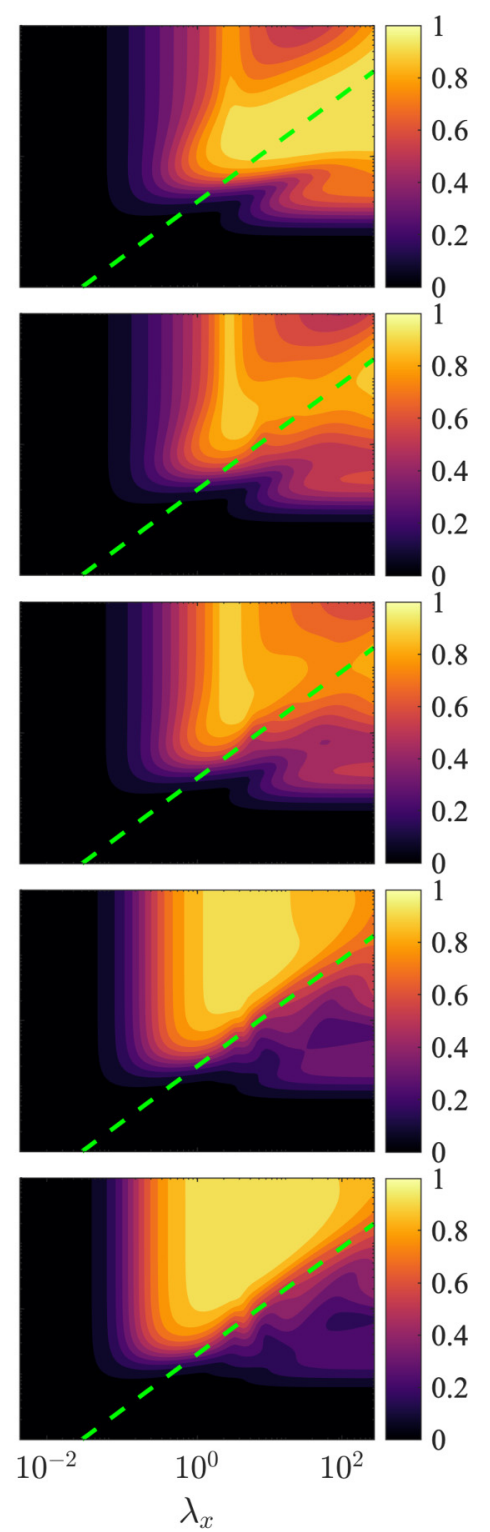

(c)

FIG. 2. Contour plots depicting the energy contained in the leading response mode relative to the total response, $\left(\sigma_{1}^{2}+\sigma_{2}^{2}\right) / \Sigma_{j} \sigma_{j}^{2}$, for different streamwise and spanwise wavelengths at (a) $c=\bar{u}\left(y^{+}=15\right)$, (b) $c=$ $\bar{u}\left(y^{+}=30\right)$, and (c) $c=\bar{u}\left(y^{+}=100\right)$ for $\mathrm{Ri}_{\tau}=0,10,18,60,100$ (top to bottom). Green dashed lines are (a) $\lambda_{x}=15 \lambda_{z}$, (b) $\lambda_{x}=10 \lambda_{z}$, and (c) $\lambda_{x}=5 \lambda_{z}$.

of two-dimensional modes for a variety of flows with strong stratification. Moreover, Mahrt [11] alludes to the emergence of two-dimensional modes (often referred to as pancake modes) owing to the conversion of vertical kinetic energy to potential energy in the presence of strong stable stratification. The premultiplied energy spectra for higher $\mathrm{Ri}_{\tau}$ indicate high energy in the vertical band as well [44]. 


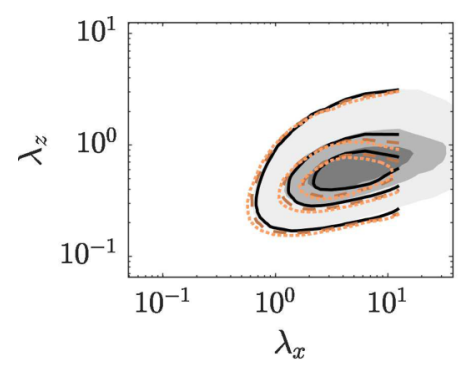

(a)

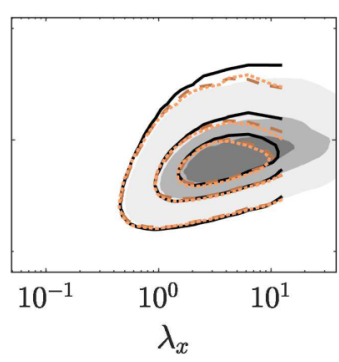

(b)

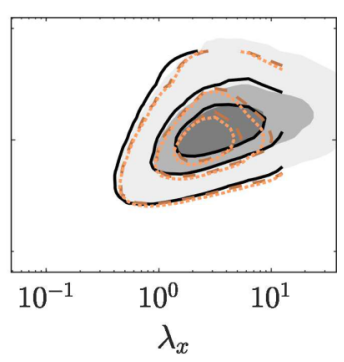

(c)

FIG. 3. Contour plots depicting the premultiplied streamwise kinetic energy spectra as functions of the streamwise and spanwise wavelengths obtained from DNS at (a) $y^{+}=15$, (b) $y^{+}=30$, and (c) $y^{+}=100$ for $\mathrm{Ri}_{\tau}=0$ (solid line), $\mathrm{Ri}_{\tau}=60$ (dashed line), and $\mathrm{Ri}_{\tau}=100$ (dotted line). The shaded contours are from the $\operatorname{Re}_{\tau}=180$ neutral channel [76]. The levels plotted are 0.1, 0.3, 0.5 times the maximum value of the corresponding spectrum.

Though not plotted here, the changes in the singular value magnitudes exhibit a similar pattern to that of Fig. 2. The largest singular values occur at larger spanwise wavelengths as stratification increases and a similar vertical band is formed for $\lambda_{x} / \delta \geqslant 1$ at $y^{+}=100$. We also see that as $\mathrm{Ri}_{\tau}$ increases the largest singular values shift from being below the green line to being above it for all of the wave speeds investigated.

\section{B. Mode shapes}

In order to study the flow structures, we compute the resolvent response modes for a set of wave parameters. The most energetic scales for the various $\mathrm{Ri}_{\tau}$ under consideration for the different wallnormal heights still coincide with the neutrally buoyant case (Fig. 3), falling in the low-rank region despite the fact that including the scalar advection-diffusion equation in the governing equations changes the wavelengths at which the resolvent operator is low-rank (Fig. 2). In this section we study the resolvent response mode shapes for these wavenumber and wave speed combinations. The list of mode combinations under consideration is listed in Table II. In particular, mode E1 is the most energetic mode for $y^{+}=15$, E2 for $y^{+}=30$, and E3 for $y^{+}=100$. To study the emergence of low-rank regions at long spanwise wavelengths we also look at the $\mathrm{S} 1$ mode at $y^{+}=100$ in Sec. III B 2.

Note that for the POD modes of the DNS, the wavenumbers in Table II are approximated such that $k_{x} L_{x} /(2 \pi)$ and $k_{z} L_{z} /(2 \pi)$ are rounded to the nearest integer. For example, for $k_{x}=\pi / 2$, $k_{x} L_{x} /(2 \pi)=\pi \sim 3$, and thus we use $k_{x}=1.5$.

TABLE II. Representative wavenumber combinations that we explore in Sec. III B.

\begin{tabular}{lccc}
\hline \hline Mode name & $k_{x}$ & $k_{z}$ & $c$ \\
\hline E1: most energetic mode for $y^{+}=15$ & $\pi / 2$ & $4 \pi$ & $\bar{u}\left(y^{+}=15\right)$ \\
E2: most energetic mode for $y^{+}=30$ & $\pi / 2$ & $3 \pi$ & $\bar{u}\left(y^{+}=30\right)$ \\
E3: most energetic mode for $y^{+}=100$ & $\pi / 2$ & $2 \pi$ & $\bar{u}\left(y^{+}=100\right)$ \\
S1: spanwise-constant mode for $y^{+}=100$ & $\pi / 2$ & 0 & $\bar{u}\left(y^{+}=100\right)$ \\
\hline \hline
\end{tabular}




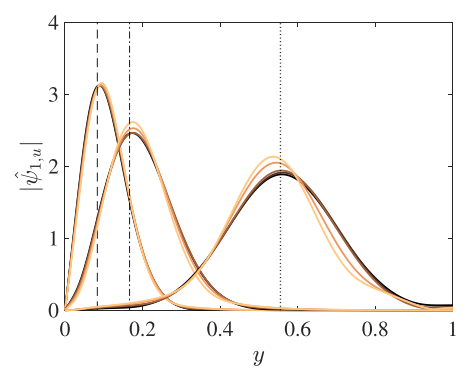

(a)

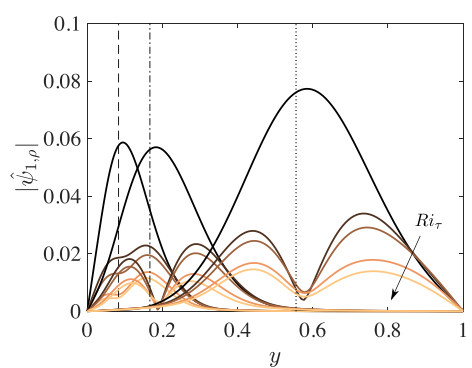

(b)

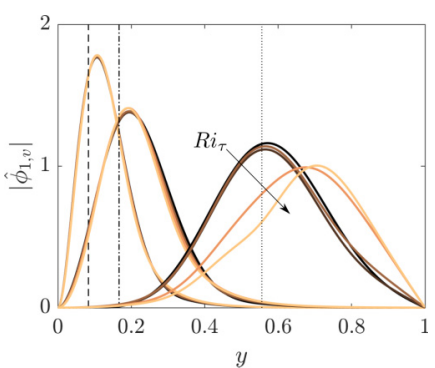

(c)

FIG. 4. Amplitudes of the leading resolvent response modes for the (a) streamwise velocity and (b) density, and leading forcing mode for the (c) wall-normal velocity for $\mathrm{Ri}_{\tau}=0,10,18,60,100$ (darker to lighter) at $c=$ $\bar{u}\left(y^{+}=15\right)$ (dashed line), $\bar{u}\left(y^{+}=30\right)$ (dot-dashed line), and $\bar{u}\left(y^{+}=100\right)$ (dotted line) for wave parameters corresponding to E1, E2, and E3, respectively. The subscripts $u$ and $\rho$ indicate the corresponding components of the resolvent response mode, and the subscript $v$ indicates the component of the forcing mode.

\section{Energy-containing modes E1, E2, and E3}

The predictive capabilities of the resolvent modes are first shown through the amplitudes of the leading resolvent response modes [Figs. 4(a) and 4(b)] of the streamwise velocity and density. The resolvent modes compare well to the streamwise and density turbulence intensities in Figs. 1(c) and 1(d). The streamwise root-mean-square (r.m.s.) quantities and resolvent amplitudes show no variation among different Richardson numbers closer to the wall and increase slightly with $\mathrm{Ri}_{\tau}$ farther away from the wall. On the other hand, the density r.m.s. and resolvent amplitudes decrease significantly with Richardson number at all wall-normal heights. Despite only using the leading resolvent mode, the relative magnitude at each corresponding wall-normal height is well captured for the range of Richardson numbers considered here. The wall-normal forcing mode component indicates that the lift-up mechanism exists even as stratification is increased; see Fig. 4. The forcing amplitudes are unaffected by the different Richardson numbers in the near-wall region, but when $y^{+}=100$ the amplitudes decrease slightly.

Additionally, we examine the response mode shapes in two dimensions for the different regions and compare the structures observed in the resolvent modes with the POD modes obtained from the DNS data. The POD modes are computed as the principal component of matrix $X$, where each row of $X$ is the wall-normal vector corresponding to the $\left(k_{x}, k_{z}\right)$ Fourier mode of the velocity and density components. The principal component is the first left singular vector of $X$, where the energy norm in Eq. (10) is used to compute the SVD. Over 1000 snapshots from DNS data are used to form matrix $X[78]$.

The two-dimensional structures of mode E1, which coincides with the size of the near the wall structures observed previously in experiments and simulations [79,80], are plotted in Fig. 5 for a reference location of $y^{\prime+}=15$. The POD modes of the streamwise, wall-normal, and spanwise velocity fields, as well as the density field, are shown in Fig. 6, for a two-dimensional slice at $\Delta z=0$. Even though the POD modes are integrated results from all values of $\omega$, we see that the POD modes are more or less centered around the $y^{+}=15$ region.

Finally, we examine the response mode shapes in two dimensions for the different regions and compare the structures observed in the resolvent modes with the autocorrelation coefficient from the DNS data. We first define the streamwise autocovariance as

$$
\hat{R}_{q q}\left(k_{x}, y, y^{\prime}, k_{z}\right)=\left\langle\hat{q}\left(k_{x}, y, k_{z}\right) \hat{q}^{*}\left(k_{x}, y^{\prime}, k_{z}\right)\right\rangle,
$$

where $q$ is a generic variable of zero mean and $\langle\cdot\rangle$ is the expected value. The autocovariance in physical space, $R_{q q}\left(\Delta x, y, y^{\prime}, \Delta z\right)$, is obtained as the inverse Fourier transform of $\hat{R}$, where 


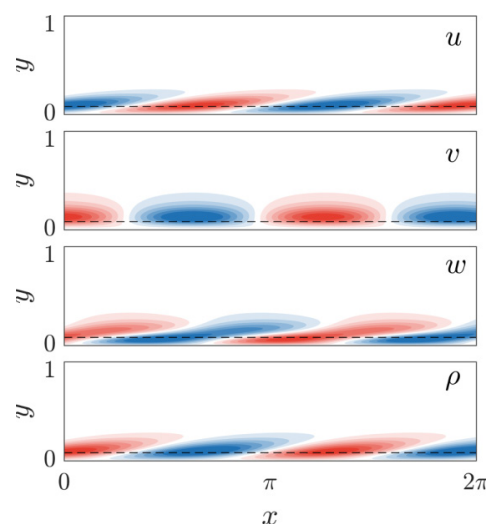

(a)

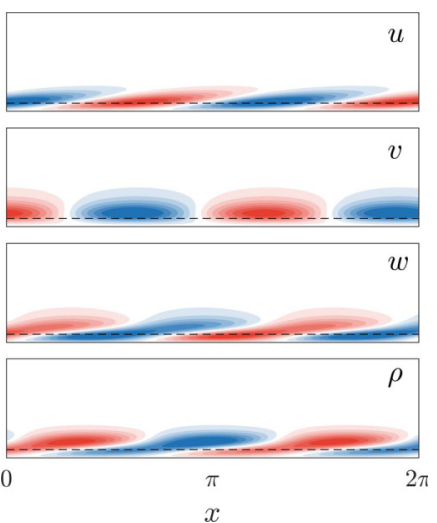

(b)

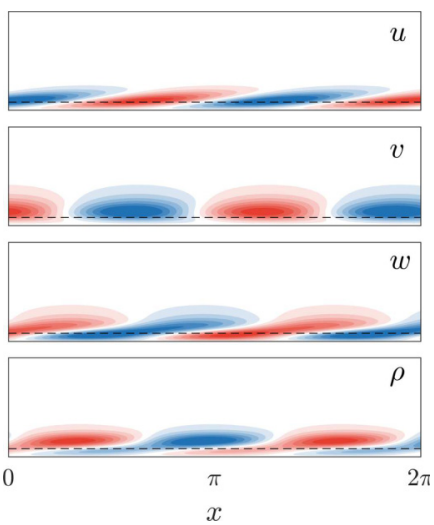

(c)

FIG. 5. Two-dimensional slices of response mode shapes for $\left(k_{x}, k_{z}\right)=(\pi / 2,4 \pi)$ at a critical-layer location of $y^{+}=15$ for (a) $\mathrm{Ri}_{\tau}=0$, (b) 18 , and (c) 100. Red and blue contours represent positive and negative fluctuations, respectively. The contour levels are scaled by the maximum of each mode component. The dashed black line in each subplot is the location of the critical layer where $c=\bar{u}\left(y^{+}=15\right)$.

$\Delta x=x-x^{\prime}$ and $\Delta z=z-z^{\prime}$ are the distances between the two points in the homogeneous directions. The autocorrelation coefficient,

$$
C_{q q}\left(\boldsymbol{x}, \boldsymbol{x}^{\prime}\right)=\frac{R_{q q}\left(\boldsymbol{x}, \boldsymbol{x}^{\prime}\right)}{\varsigma_{q}(\boldsymbol{x}) \zeta_{q}\left(\boldsymbol{x}^{\prime}\right)},
$$

is obtained by normalizing the covariance with the product of the standard deviations, $\varsigma$, at the two points involved in the measurements, which is the normalization adopted by most researchers [81-86].

The two-dimensional structures of mode E1, which coincides with the size of the near the wall structures observed previously in experiments and simulations [79,80], are plotted in Fig. 5. The autocorrelations of the streamwise, wall-normal, and spanwise velocity fields as well as the density field are shown in Fig. 7, for a two-dimensional slice at $\Delta z=0$. The reference location $y^{\prime+}=15$.

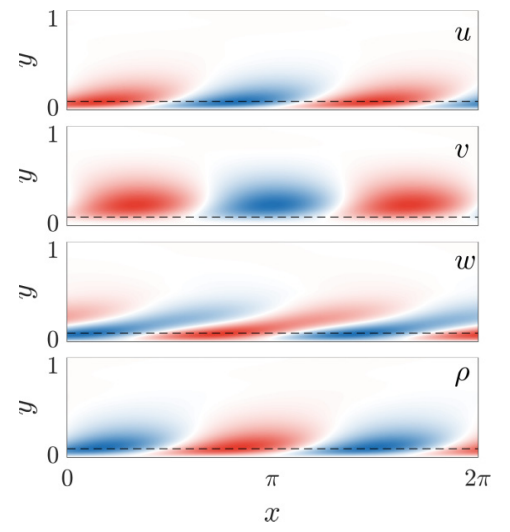

(a)

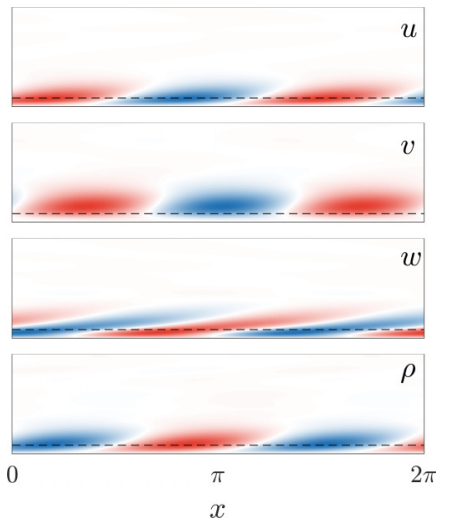

(b)

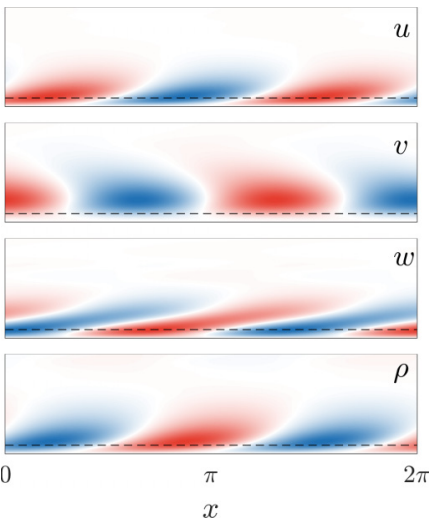

(c)

FIG. 6. POD modes corresponding to $\left(k_{x}, k_{z}\right)=(\pi / 2,4 \pi)$ for (a) $\mathrm{Ri}_{\tau}=0$, (b) 18 , and (c) 100 . The horizontal dashed line is $y^{+}=15$. 


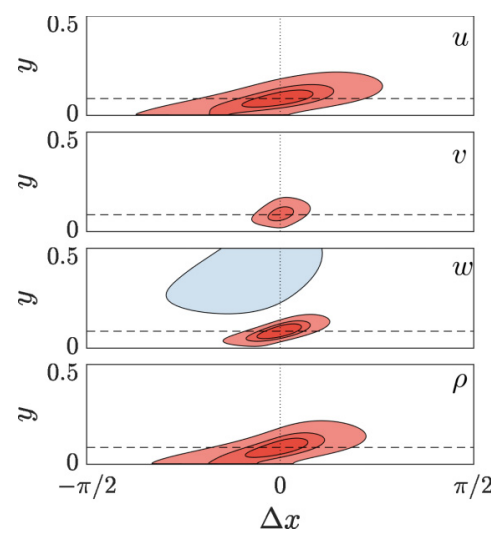

(a)

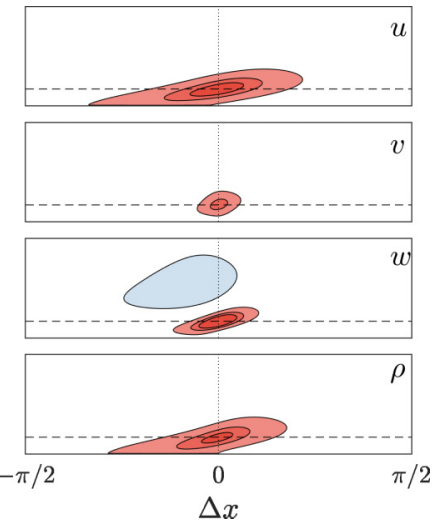

(b)

FIG. 7. Autocorrelation coefficients $C_{u u}, C_{v v}, C_{w w}$, and $C_{\rho \rho}$ of the DNS at $y^{+}=15$ for (a) $\mathrm{Ri}_{\tau}=0$ and (b) 100. Red and blue contours represent positive $(0.4,0.6,0.8)$ and negative $(-0.2)$ correlation, respectively, with each contour level signifying 0.2 increments. The horizontal dashed line is $y^{+}=15$, and the vertical dotted line is $\Delta x=0$.

The LES of Ref. [35] and the DNS of Ref. [44] demonstrated that structures in the near-wall region $\left(y^{+} \lesssim 15\right)$ are largely unaffected by stable stratification. As expected, both the resolvent response modes and the correlations do not change significantly for the range of $\mathrm{Ri}_{\tau}$ considered. For the velocities, the main difference is a slight backward tilt in the wall-normal component. The largest difference occurs for density properties as the phase in the wall-normal direction along the resolvent response modes are shifted, creating structures that are more detached from the wall. Similarly, the density POD modes and the correlations shift farther away from the wall for higher values of $\mathrm{Ri}_{\tau}$.

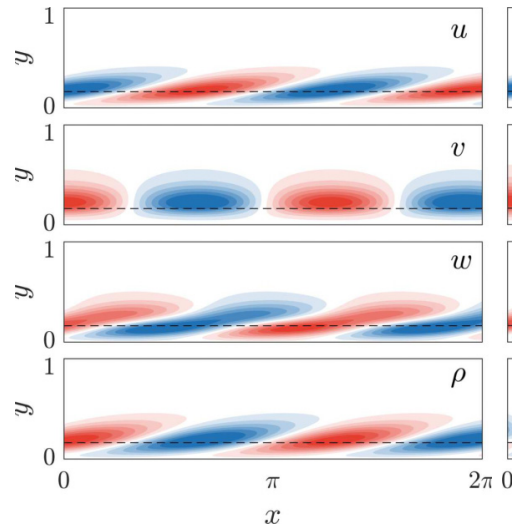

(a)

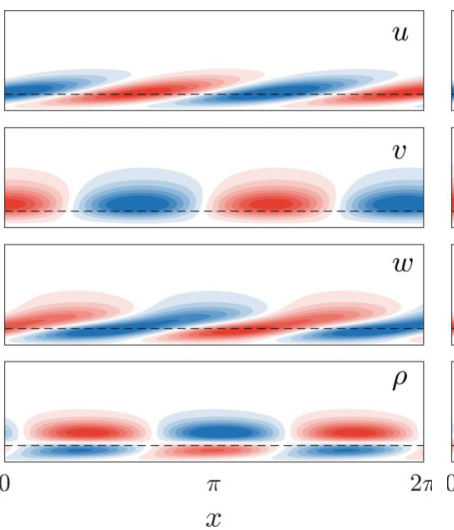

(b)

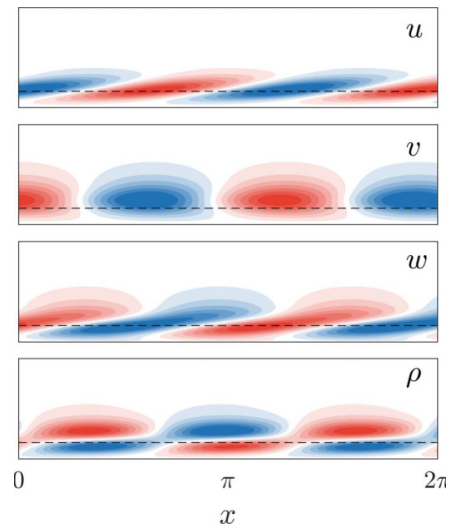

(c)

FIG. 8. Two-dimensional slices of response mode shapes for $\left(k_{x}, k_{z}\right)=(\pi / 2,3 \pi)$ at a critical-layer location of $y^{+}=30$ for (a) $\mathrm{Ri}_{\tau}=0$, (b) 18 , and (c) 100. Red and blue contours represent positive and negative fluctuations, respectively. The contour levels are scaled by the maximum of each mode component. The dashed black line in each subplot is the location of the critical layer where $c=\bar{u}\left(y^{+}=30\right)$. 


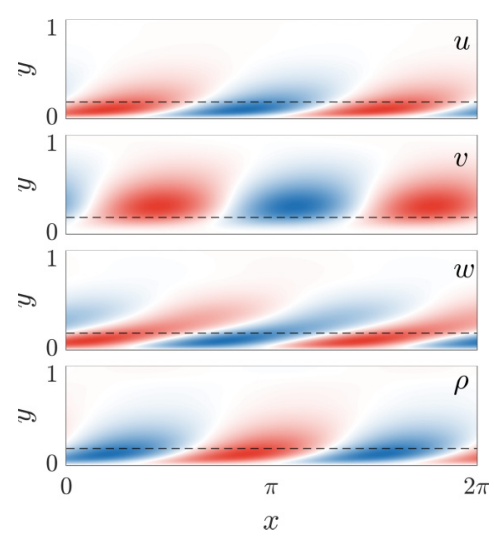

(a)

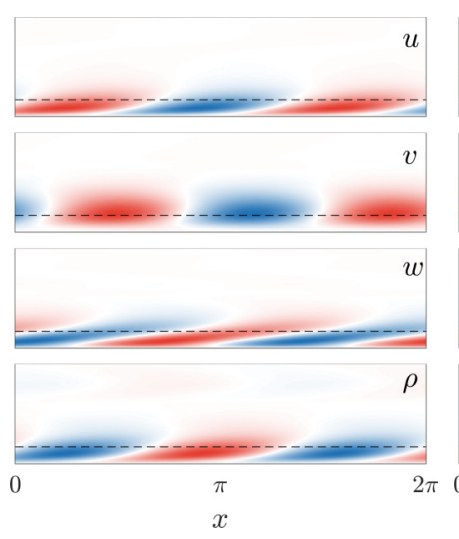

(b)

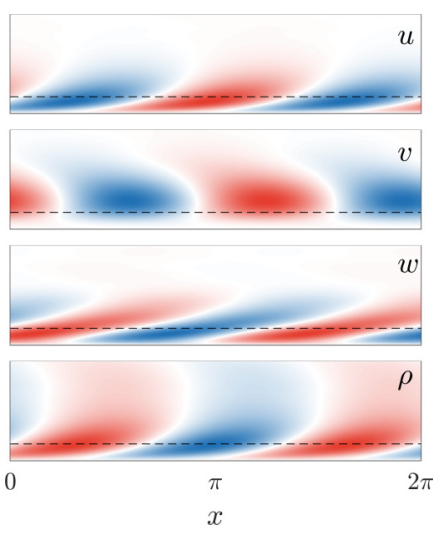

(c)

FIG. 9. POD modes corresponding to $\left(k_{x}, k_{z}\right)=(\pi / 2,3 \pi)$ for (a) $\mathrm{Ri}_{\tau}=0$, (b) 18 , and (c) 100 . The horizontal dashed line is $y^{+}=30$.

We plot the resolvent response modes (Fig. 8) and the POD modes (Fig. 9) for the wavenumbers and wave speed corresponding to E2. The results are similar to that of E1, since the velocity response modes do not vary significantly across $\mathrm{Ri}_{\tau}$, but a difference is observed in the density modes as a phase change along $y$. The POD modes for density are both wall-detached in the $\mathrm{Ri}_{\tau}=0$ and $\mathrm{Ri}_{\tau}=100$ case, although the center of the density POD modes for the $\mathrm{Ri}_{\tau}=100$ case lies farther away from the wall.

The biggest difference in the resolvent response modes for the different Richardson numbers can be seen for the wavenumber and wave speed corresponding to E3. We plot the resolvent response modes (Fig. 10) and the POD modes (Fig. 11) for the wavenumbers and wave speed corresponding to E3. Here all resolvent modes show significant differences in the stratified case compared to the unstratified case. In particular, the backwards tilting of the velocity modes and the phase difference across $y$ of the density mode are pronounced. These phenomena occur in the

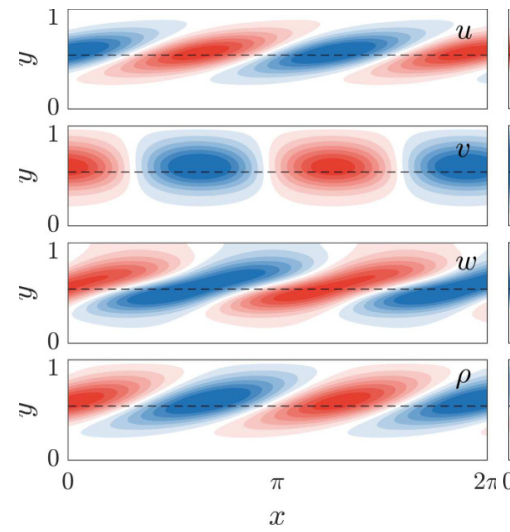

(a)

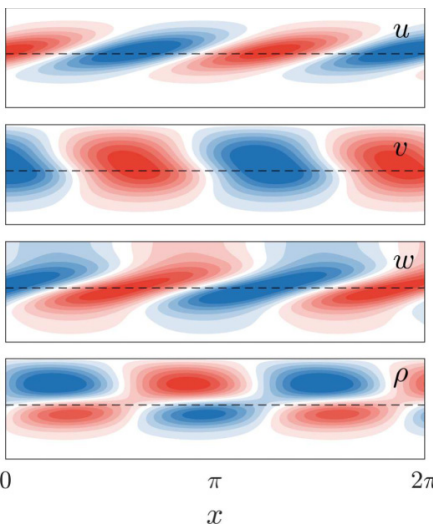

(b)

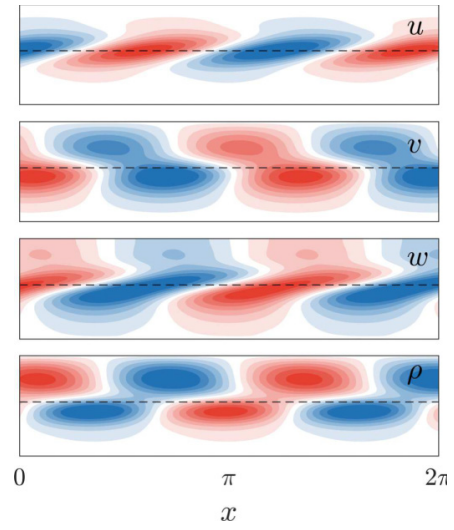

(c)

FIG. 10. Two-dimensional slices of response mode shapes for $\left(k_{x}, k_{z}\right)=(\pi / 2,2 \pi)$ at a critical-layer location of $y^{+}=100$ for (a) $\mathrm{Ri}_{\tau}=0$, (b) 18, and (c) 100. Red and blue contours represent positive and negative fluctuations, respectively. The contour levels are scaled by the maximum of each mode component. The dashed black line in each subplot is the location of the critical layer where $c=\bar{u}\left(y^{+}=100\right)$. 


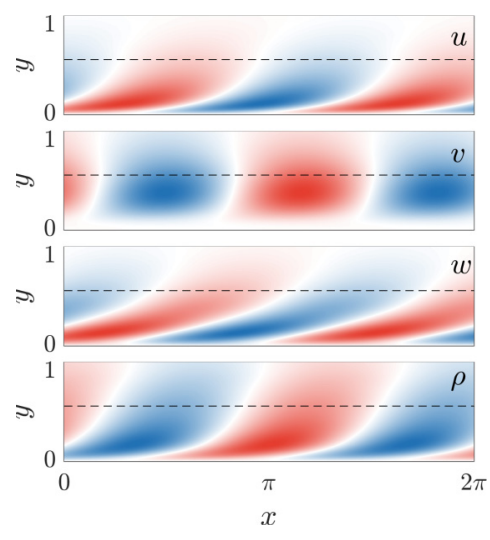

(a)

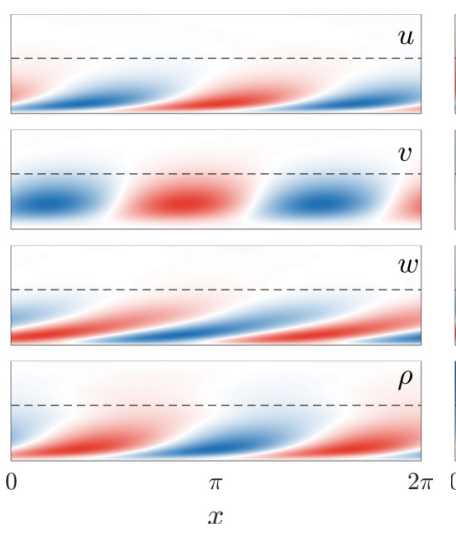

(b)

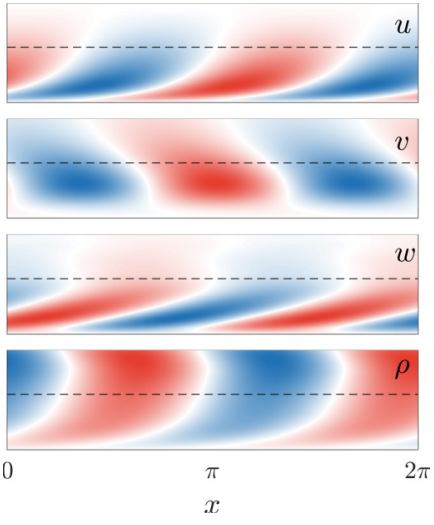

(c)

FIG. 11. POD modes corresponding to $\left(k_{x}, k_{z}\right)=(\pi / 2,2 \pi)$ for (a) $\mathrm{Ri}_{\tau}=0$, (b) 18, and (c) 100. The horizontal dashed line is $y^{+}=100$.

POD modes as well. There is noticeable backwards tilting in the all three velocity components with particularly noticeable changes in the wall-normal POD mode and shift in the density POD modes in the $\mathrm{Ri}_{\tau}=100$ case compared to the neutrally stratified case. The biggest differences come in the form of the wall-normal and density models because they are coupled through the Richardson number in the stratified Navier-Stokes equations.

\section{Spanwise-constant mode S1}

As stratification is increased the low-rank behavior of the resolvent operator shifts from moderate-to-large streamwise wavelengths to moderate-to-large spanwise wavelengths (Fig. 2). Here we explore the amplitude and structure of the S1 mode combination given in Table II.

First, we observe the leading response mode and forcing amplitudes in Fig. 12. We see that increasing the stratification decreases the streamwise and density response mode amplitude significantly. Interestingly, the streamwise response now has a double-peaked structure and the density response does not; this is opposite to the mode shapes observed for the E3 mode at $y^{+}=100$

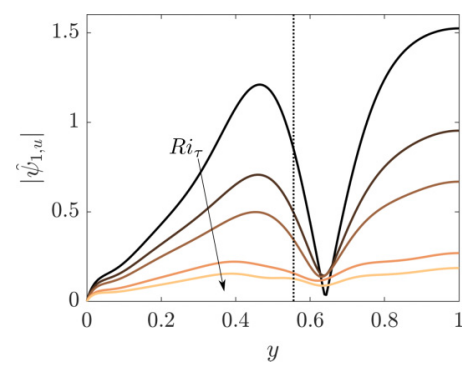

(a)

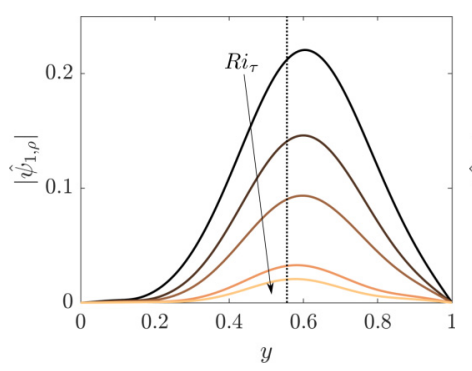

(b)

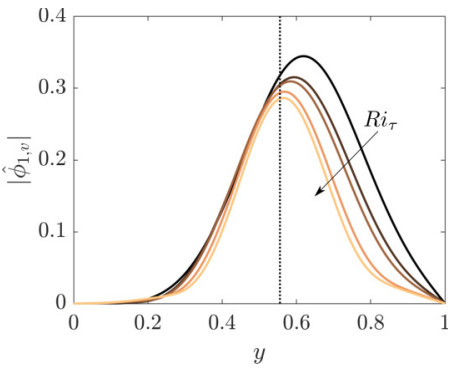

(c)

FIG. 12. Amplitudes of the leading resolvent response modes for the (a) streamwise velocity and (b) density, and leading forcing mode for the (c) wall-normal velocity for $\mathrm{Ri}_{\tau}=0,10,18,60,100$ (darker to lighter) at $c=\bar{u}\left(y^{+}=100\right)$ (dotted line) for wave parameters corresponding to $\mathrm{S} 1$, respectively. The subscripts $u$ and $\rho$ indicate the corresponding components of the resolvent response mode, and the subscript $v$ indicates the component of the forcing mode. 


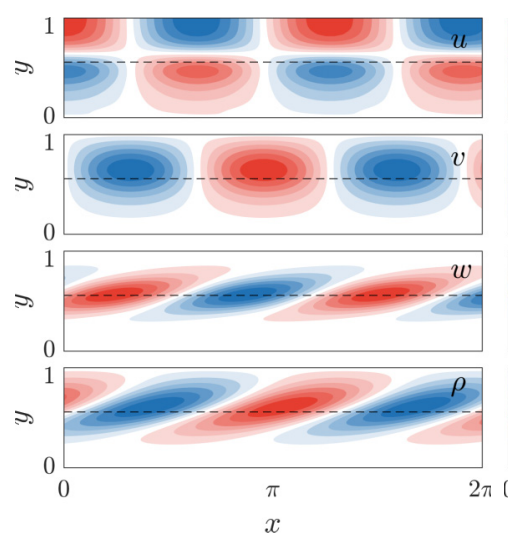

(a)

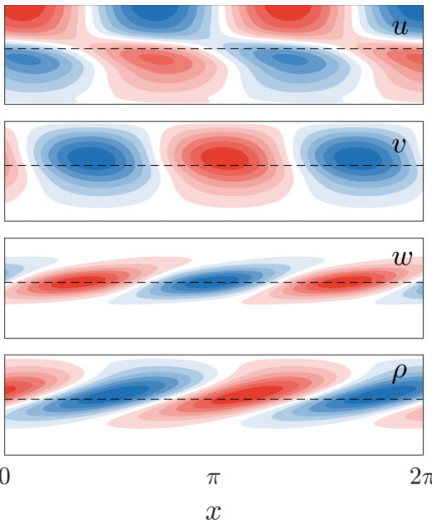

(b)

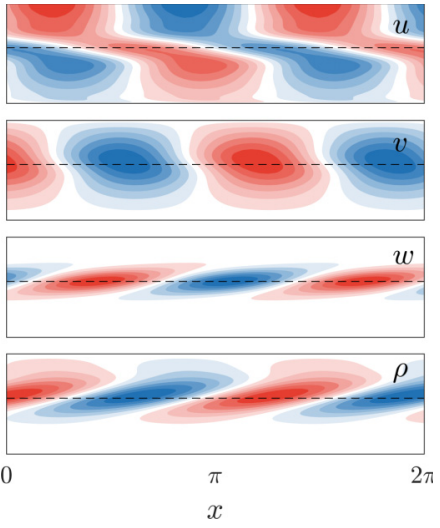

(c)

FIG. 13. Two-dimensional slices of response mode shapes the long-in-spanwise mode (S1) where $\left(k_{x}, k_{z}\right)=(\pi / 2, \pi / 20)$ at a critical-layer location of $y^{+}=100$ for (a) $\operatorname{Ri}_{\tau}=0$, (b) 18 , and (c) 100 . Red and blue contours represent positive and negative fluctuations, respectively. The contour levels are scaled by the maximum of each mode component. The dashed black line in each subplot is the location of the critical layer where $c=\bar{u}\left(y^{+}=100\right)$.

in Fig. 4. For the $\mathrm{S} 1$ mode, the amplitude of the wall-normal forcing component is reduced with stratification and is significantly lower than the E3 wall-normal forcing amplitude shown in Fig. 4(c).

The two-dimensional resolvent response mode and POD modes for S1 are shown in Figs. 13 and 14, respectively. Here all resolvent modes show differences in the stratified case compared to the unstratified case. In particular, the backwards tilting of the streamwise and wall-normal velocity modes. The spanwise and density components become more localized to the critical layer at $y^{+}=$ 100. The POD modes also show the backward tilt in the streamwise and wall-normal component. The biggest difference between the POD and resolvent modes occur in the density component. The density POD mode gets further from the wall with stratification and is centered above the critical layer.

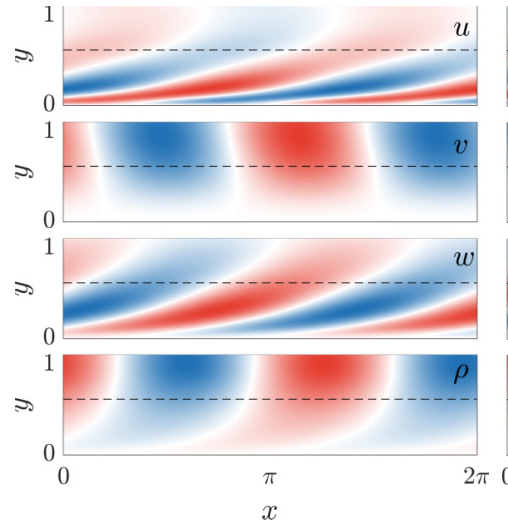

(a)

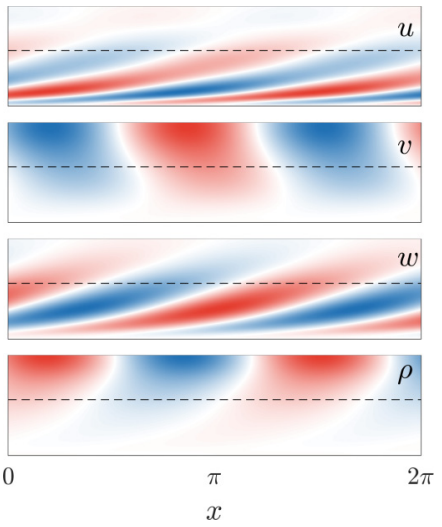

(b)

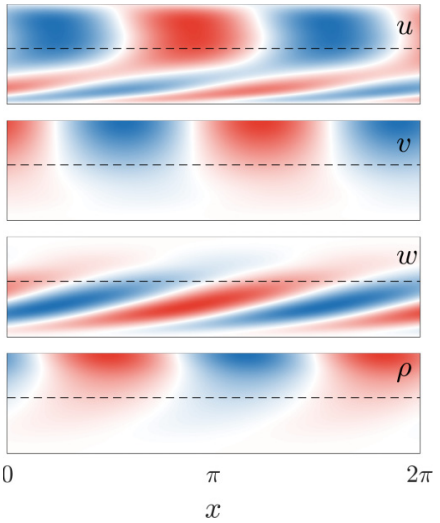

(c)

FIG. 14. POD modes corresponding to $\left(k_{x}, k_{z}\right)=(\pi / 2,0)$ for (a) $\mathrm{Ri}_{\tau}=0$, (b) 18 , and (c) 100 . The horizontal dashed line is $y^{+}=100$. 


\section{Energy balance at selected scales}

Finally, we study the energy budget terms for the turbulent kinetic energy of the stratified channel. We define the production, transport, viscous diffusion/dissipation, buoyancy flux, and pressurestrain budget terms in the resolvent formulation $[66,87]$ as

$$
\begin{aligned}
& \mathcal{P}_{\text {tot }}(y)=\mathbb{R}\left[-\frac{\partial \bar{u}}{\partial y} \sum_{j} \int_{-\infty}^{\infty} \sigma_{j}^{2} \chi_{j}^{2}\left(\hat{\boldsymbol{\psi}}_{j, u}^{*} \hat{\boldsymbol{\psi}}_{j, v}\right) d \boldsymbol{k}\right], \\
& \mathcal{T}_{\text {tot }}(y)=\mathbb{R}\left[-\sum_{j} \sum_{i} \int_{-\infty}^{\infty} \sigma_{j} \chi_{j} \chi_{i}\left(\hat{\boldsymbol{\phi}}_{i, u}^{*} \hat{\boldsymbol{\psi}}_{j, u}+\hat{\boldsymbol{\phi}}_{i, v}^{*} \hat{\boldsymbol{\psi}}_{j, v}+\hat{\boldsymbol{\phi}}_{i, w}^{*} \hat{\boldsymbol{\psi}}_{j, w}\right) d \boldsymbol{k}\right], \\
& \mathcal{V}_{\text {tot }}(y)=\mathbb{R}\left[\frac{1}{\operatorname{Re}_{\tau}} \sum_{j} \int_{-\infty}^{\infty} \sigma_{j}^{2} \chi_{j}^{2}\left(\hat{\boldsymbol{\psi}}_{j, u}^{*} \hat{\Delta} \hat{\boldsymbol{\psi}}_{j, u}+\hat{\boldsymbol{\psi}}_{j, v}^{*} \hat{\Delta}_{\hat{\boldsymbol{\psi}}} \hat{\boldsymbol{\psi}}_{j, v}+\hat{\boldsymbol{\psi}}_{j, w}^{*} \hat{\Delta} \hat{\boldsymbol{\psi}}_{j, w}\right) d \boldsymbol{k}\right], \\
& \mathcal{B}_{\text {tot }}(y)=\mathbb{R}\left[-\operatorname{Ri}_{\tau} \sum_{j} \int_{-\infty}^{\infty} \sigma_{j}^{2} \chi_{j}^{2}\left(\hat{\boldsymbol{\psi}}_{j, v}^{*} \hat{\boldsymbol{\psi}}_{j, \rho}\right) d \boldsymbol{k}\right], \\
& \Pi_{\mathrm{tot}}(y)=\mathbb{R}\left[-\sum_{j} \int_{-\infty}^{\infty} \sigma_{j}^{2} \chi_{j}^{2} D_{y}\left(\hat{\boldsymbol{\psi}}_{j, p}^{*} \hat{\boldsymbol{\psi}}_{j, v}\right) d \boldsymbol{k}\right],
\end{aligned}
$$

where $\chi_{j}, \sigma_{j}, \hat{\boldsymbol{\psi}}_{j}$, and $\hat{\boldsymbol{\phi}}_{j}$ are functions of $\boldsymbol{k}$ and the subscripts $u, v, w$, and $\rho$ indicate the corresponding components of the response or forcing mode. To get a global sense of the energy balance, the equations above are integrated over all wavenumber triplets. Here we will examine only the principal resolvent mode contribution to the local components of the total budgets for particular $\boldsymbol{k}$, defined as

$$
\begin{aligned}
& \mathcal{P}(y, \boldsymbol{k})=\mathbb{R}\left[-\frac{\partial \bar{u}}{\partial y} \sigma_{1}^{2}\left(\hat{\boldsymbol{\psi}}_{1, u}^{*} \hat{\boldsymbol{\psi}}_{1, v}\right)\right], \\
& \mathcal{T}(y, \boldsymbol{k})=\mathbb{R}\left[-\sigma_{1}\left(\hat{\boldsymbol{\phi}}_{1, u}^{*} \hat{\boldsymbol{\psi}}_{1, u}+\hat{\boldsymbol{\phi}}_{1, v}^{*} \hat{\boldsymbol{\psi}}_{1, v}+\hat{\boldsymbol{\phi}}_{1, w}^{*} \hat{\boldsymbol{\psi}}_{1, w}\right)\right], \\
& \mathcal{V}(y, \boldsymbol{k})=\mathbb{R}\left[\frac{1}{\operatorname{Re}_{\tau}} \sigma_{1}^{2}\left(\hat{\boldsymbol{\psi}}_{1, u}^{*} \hat{\Delta} \hat{\boldsymbol{\psi}}_{1, u}+\hat{\boldsymbol{\psi}}_{1, v}^{*} \hat{\Delta} \hat{\boldsymbol{\psi}}_{1, v}+\hat{\boldsymbol{\psi}}_{1, w}^{*} \hat{\Delta} \hat{\boldsymbol{\psi}}_{1, w}\right)\right] . \\
& \mathcal{B}(y, \boldsymbol{k})=\mathbb{R}\left[-\operatorname{Ri}_{\tau} \sigma_{1}^{2}\left(\hat{\boldsymbol{\psi}}_{1, v}^{*} \hat{\boldsymbol{\psi}}_{1, \rho}\right)\right], \\
& \Pi(y, \boldsymbol{k})=\mathbb{R}\left[-\sigma_{1}^{2} D_{y}\left(\hat{\boldsymbol{\psi}}_{1, p}^{*} \hat{\boldsymbol{\psi}}_{1, v}\right)\right] .
\end{aligned}
$$

The results for wavenumber combinations E1, E2, and E3 are shown in Fig. 15. Since the wavenumber combinations E1, E2, and E3 are the most energetic at each wave speed, we predict that the local components of the budget term should indicate the overall trend of the total budget term at the corresponding wall-normal height. These quantities are compared to the energy budget computed from the DNS, shown in Fig. 16. The trends observed in the energy budget computed from the DNS are also recovered in the resolvent budgets. The production is mostly balanced by viscous diffusion/dissipation and has larger magnitudes compared to the transport and pressure strain (approximately 10\% of the production term) or buoyancy flux (approximately $0.1 \%-1 \%$, depending on $\mathrm{Ri}_{\tau}$ of the production term) terms. Comparing the quantities at the wall-normal heights of interest, we see that at $y^{+}=15$, there is little variation in the production and viscous diffusion/dissipation terms in both DNS and resolvent modes. The difference in relative magnitude over the various values of $\mathrm{Ri}_{\tau}$ increases farther away from the wall, and at $y^{+}=100$, the production (and viscous diffusion/dissipation) of the $\mathrm{Ri}_{\tau}=100$ case is half the production (and viscous diffusion/dissipation) of the neutrally buoyant case in both the DNS and resolvent. 
(a)

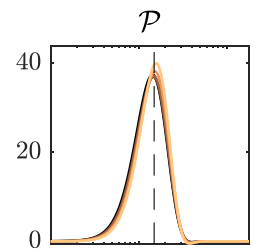

(b)

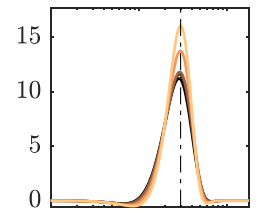

(c)

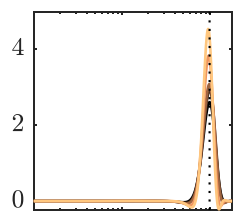

(d)

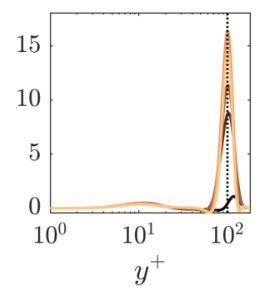

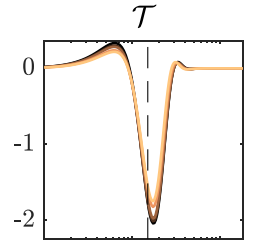
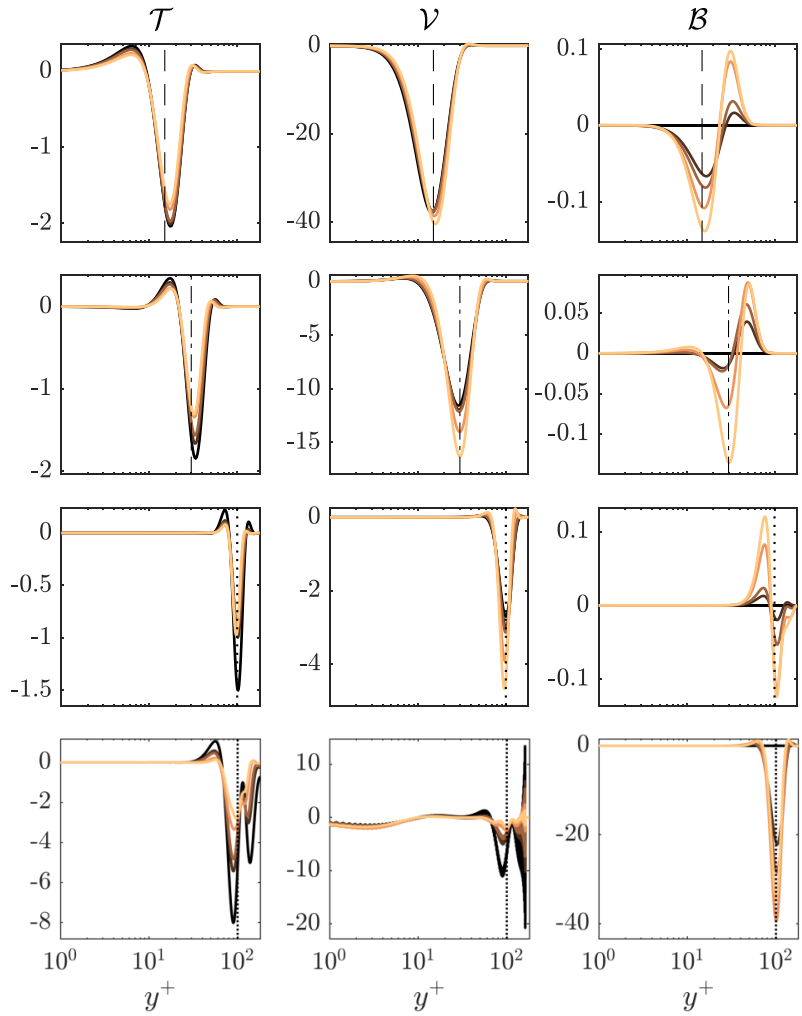
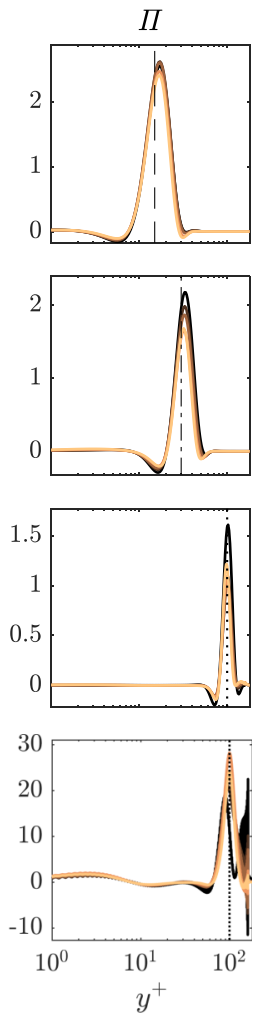

FIG. 15. Energy budget terms (production, $\mathcal{P}$; transport, $\mathcal{T}$; viscous diffusion/dissipation, $\mathcal{V}$; buoyancy flux, $\mathcal{B}$; and pressure strain, П) computed from resolvent modes [Eqs. (18a)-(18e)] for wavenumbers given by (a) E1 at $c=\bar{u}\left(y^{+}=15\right)$, (b) E2 at $c=\bar{u}\left(y^{+}=30\right)$, (c) E3, and (d) $\mathrm{S} 1$ at $c=\bar{u}\left(y^{+}=100\right.$ ) for $\mathrm{Ri}_{\tau}=$ $0,10,18,60,100$ (darker to lighter).

For the S1 modes, the amplitudes of the production and viscous diffusion/dissipation terms are significantly smaller than the E1, E2, and E3 wavenumber combinations. It is also evident that production increases with stratification, and viscous diffusion/dissipation is almost entirely reduced.

Direct comparison of the integrated magnitudes is more difficult for the transport, pressure strain, and buoyancy flux terms as they are not uniformly positive or negative. However, this indicates
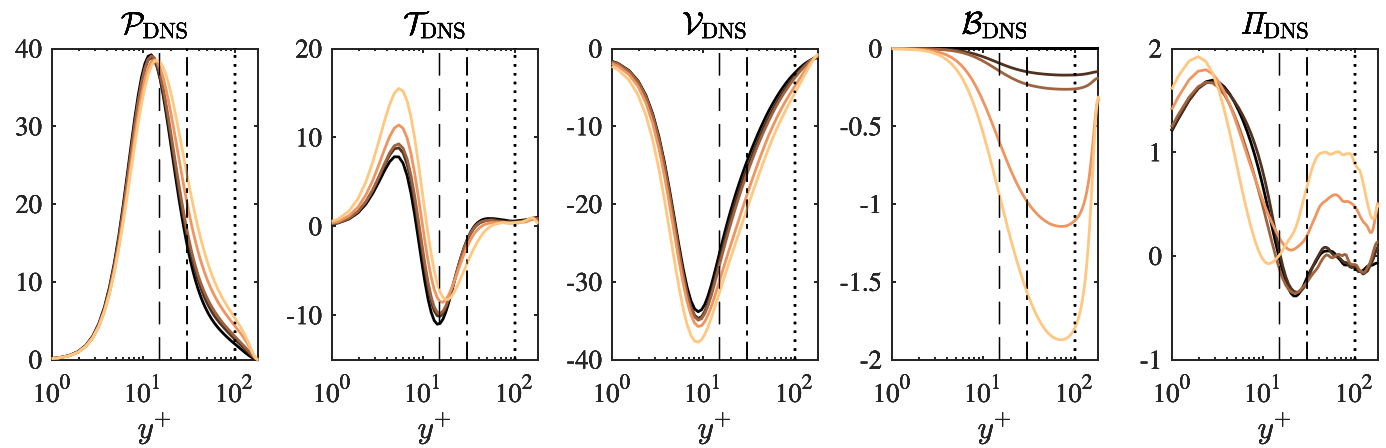

FIG. 16. (Energy budget terms computed from the $\mathrm{DNS}$ for $\mathrm{Ri}_{\tau}=0,10,18,60,100$ (darker to lighter). 


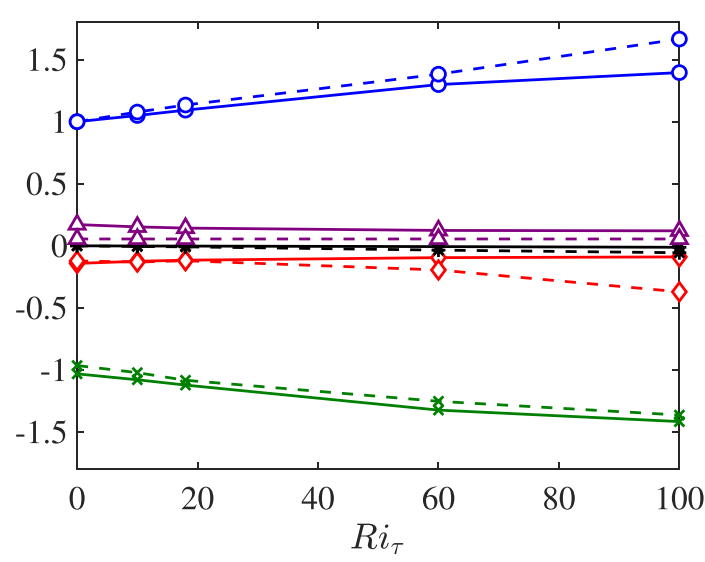

(a)

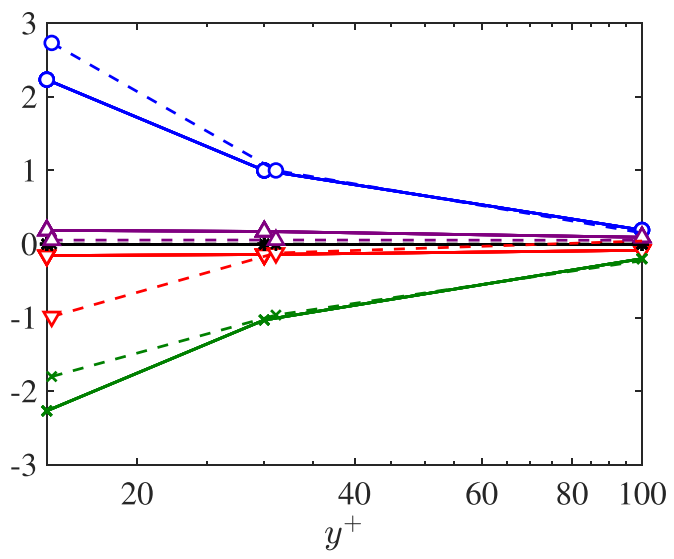

(b)

FIG. 17. (a) Resolvent (solid line) and DNS (dashed line) energy budget terms normalized by the respective $\mathcal{P}\left(\mathrm{Ri}_{\tau}=0, y^{+}=30\right.$ ) as a function of $\mathrm{Ri}_{\tau}$ for $y^{+}=30$. (b) Resolvent (solid line) and DNS (dashed line) energy budget terms normalized by the respective $\mathcal{P}\left(\mathrm{Ri}_{\tau}=0, y^{+}=30\right)$ as a function of $y^{+}$for $\mathrm{Ri}_{\tau}=0$. Lines indicate production (blue, circles), transport (red, diamonds), viscous diffusion/dissipation (green, crosses), buoyancy flux (black, asterisks), and pressure strain (purple, triangles).

that, locally, buoyancy flux acts as an energy transfer term, much like the turbulent transport, as the term adds energy in one wall-normal location and removes it from another. Because the DNS energy budget is integrated for all spatiotemporal scales, it is impossible to deduce that the buoyance flux term acts as a local energy transfer term from Fig. 16, which shows a net negative energy balance from $\mathcal{B}$ at all wall-normal locations. In contrast, the resolvent buoyancy flux term indicates a nonmonotonic distribution of energy in the wall-normal direction. Similar results could be obtained through spatiotemporal deconstruction of the DNS energy budget term as in Ref. [88], but this would require a time-resolved data set for a longer time domain. The resolvent turbulent transport and pressure-strain terms stay relatively similar among different $\mathrm{Ri}_{\tau}$, as does the turbulent transport and pressure-strain terms from DNS. The buoyancy flux is much more dependent on $\mathrm{Ri}_{\tau}$, with variations becoming greater farther away from the wall in both the DNS and resolvent results.

These results can be better quantified by plotting the values at each wall-normal location normalized by the production at $y^{+}=15$ for the $\mathrm{Ri}_{\tau}=0$ case for both the resolvent and the DNS energy budgets, as shown in Fig. 17. This shows that the overall trend of the budget terms is well captured by the resolvent budget terms, as a function of both friction Richardson number and wall-normal location, with the exception of the transport term close to the wall. This discrepancy may be attenuated by integrating over more wave speeds.

Note that the results are not expected to match that of DNS for all scales as the the energy captured in the wall-parallel resolvent modes is known to be overpredicted and the energy captured in the Reynolds stress and wall-normal resolvent modes underpredicted. This is a known issue for the resolvent analysis in the primitive variables due to the competing mechanisms of the Squire modes with the Orr-Sommerfeld modes [89,90]. Additionally, the underprediction of energy captured in the Reynolds stress and wall-normal resolvent modes could explain the underprediction of the transport term close to the wall. Crucially, though, the most energetic scale can reproduce the integrated effect of all scales, which enables a quick predictive model of stratified boundary layers. The discrepancy in the energy prediction between the resolvent model and DNS could be reconciled by using nonlinear forcing terms computed from DNS as inputs to the resolvent model as shown in Refs. [61,64]. 


\section{CONCLUSIONS}

The resolvent framework for the Navier-Stokes equations with the Boussinesq approximation was applied to a stratified turbulent boundary layer. Computation of the leading resolvent modes is more cost-effective than performing a full-scale simulation or experiment, while being able to provide information on the flow. This quick model can provide meaningful insight into stratified flows with only information about the mean profile and prior knowledge of energetic scales of motion in the neutrally buoyant boundary layers.

The results show that despite using only a very limited range of representative scales, the resolvent model was able to reproduce the relative magnitude of turbulence intensities and the balance of the energy budget as well as provide meaningful analysis of structures in the flow. We studied the amplitude of the resolvent response modes and two-dimensional slices of the mode shapes of the rank-one approximation, which were then compared to the turbulence intensities and the two-dimensional autocorrelation of the velocity and density fields of the DNS, respectively. The resolvent response modes were able to predict the relative variation in turbulence intensities as a function of wall-normal distance and Richardson number for the $\mathrm{Ri}_{\tau}$ under consideration in this study. The two-dimensional mode shapes also provided insight into how the autocorrelation coefficient might shift as a function of $\mathrm{Ri}_{\tau}$. Finally, the energy budget terms for the turbulent kinetic energy of the system were computed both using the rank-one approximation of the resolvent analysis and the DNS data. Again, the resolvent energy budget predicts well the relative distribution of energy between production, dissipation, transport, and buoyancy flux as a function of wall-normal distance and Richardson number.

In the current study, the resolvent model was closed using mean velocity and density profiles obtained from DNS and using the assumption of white-noise forcing. For full closure of the model we would need to model the nonlinear forcing term or calculate it using data from simulations or experiments. This was not explored in the present investigation but remains a topic of future work. The computational cost of calculating the forcing and response modes at certain scales was on the order of seconds on a laptop. Therefore, by obtaining only mean velocity and scalar profiles we could generate a salient basis for a given stratified wall-bounded flow. The next steps involve using in situ data to generate modes that are representative of flow phenomena observed in nature.

\section{ACKNOWLEDGMENTS}

The support of a Vannevar Bush Faculty Fellowship administered under the US Office of Naval Research, Grant No. N00014-17-1-3022, is gratefully acknowledged. Additionally, the authors would like to thank Dr. Angeliki Laskari for insightful discussions.

[1] F. T. M. Nieuwstadt, The turbulent structure of the stable, nocturnal boundary layer, J. Atm. Sci. 41, 2202 (1984).

[2] R. B. Stull, Meteorology for Scientists and Engineers (Brooks/Cole, Pacific Grove, CA, 2000).

[3] C. Wunsch and R. Ferrari, Vertical mixing, energy, and the general circulation of the oceans, Annu. Rev. Fluid Mech. 36, 281 (2004).

[4] S. A. Thorpe, The Turbulent Ocean (Cambridge University Press, Cambridge, 2005).

[5] G. N. Ivey, K. B. Winters, and J. R. Koseff, Density stratification, turbulence, but how much mixing? Annu. Rev. Fluid Mech. 40, 169 (2008).

[6] H. A. Panofsky and J. A. Dutton, Atmospheric Turbulence: Models and Methods for Engineering Applications (Wiley, 1984). 
[7] Z. Sorbjan, Structure of the Atmospheric Boundary Layer (Prentice Hall, 1989).

[8] R. B. Stull, An Introduction to Boundary Layer Meteorology, Atmospheric and Oceanographic Sciences Library, Vol. 13 (Springer Science \& Business Media, 1988).

[9] J. C. Wyngaard, Turbulence in the Atmosphere (Cambridge University Press, 2010).

[10] J. R. Garratt, The atmospheric boundary layer, Earth-Sci. Rev. 37, 89 (1994).

[11] L. Mahrt, Stably stratified atmospheric boundary layers, Annu. Rev. Fluid Mech. 46, 23 (2014).

[12] L. Mahrt, Stratified atmospheric boundary layers, Bound.-Layer Meteorol. 90, 375 (1999).

[13] O. Williams, T. Hohman, T. Van Buren, E. Bou-Zeid, and A. J. Smits, The effect of stable thermal stratification on turbulent boundary layer statistics, J. Fluid Mech. 812, 1039 (2017).

[14] K. Chauhan, N. Hutchins, J. Monty, and I. Marusic, Structure inclination angles in the convective atmospheric surface layer, Bound.-Layer Meteorol. 147, 41 (2013).

[15] S. T. Salesky and W. Anderson, Buoyancy effects on large-scale motions in convective atmospheric boundary layers: Implications for modulation of near-wall processes, J. Fluid Mech. 856, 135 (2018).

[16] S. T. Salesky and W. Anderson, Revisiting inclination of large-scale motions in unstably stratified channel flow, J. Fluid Mech. 884, R5 (2020).

[17] J. R. Taylor and S. Sarkar, Internal gravity waves generated by a turbulent bottom ekman layer, J. Fluid Mech. 590, 331 (2007).

[18] J. C. Wyngaard and O. R. Coté, The budgets of turbulent kinetic energy and temperature variance in the atmospheric surface layer, J. Atm. Sci. 28, 190 (1971).

[19] J. Kondo, O. Kanechika, and N. Yasuda, Heat and momentum transfers under strong stability in the atmospheric surface layer, J. Atm. Sci. 35, 1012 (1978).

[20] L. Mahrt, Nocturnal boundary-layer regimes, Bound.-Layer Meteorol. 88, 255 (1998).

[21] H. J. S. Fernando and J. C. Weil, Whither the stable boundary layer? A shift in the research agenda, Bull. Am. Meteorol. Soc. 91, 1475 (2010).

[22] A.-S. Smedman, M. Tjernström, and U. Högström, The near-neutral marine atmospheric boundary layer with no surface shearing stress: A case study, J. Atm. Sci. 51, 3399 (1994).

[23] M. T. Stacey, S. G. Monismith, and J. R. Burau, Observations of turbulence in a partially stratified estuary, J. Phys. Oceanogr. 29, 1950 (1999).

[24] C. E. Bluteau, N. L. Jones, and G. N. Ivey, Dynamics of a tidally-forced stratified shear flow on the continental slope, J. Geophys. Res.: Oceans 116 (2011).

[25] Y. Lu, R. G. Lueck, and D. Huang, Turbulence characteristics in a tidal channel, J. Phys. Oceanogr. 30, 855 (2000).

[26] K. A. Davis and S. G. Monismith, The modification of bottom boundary layer turbulence and mixing by internal waves shoaling on a barrier reef, J. Phys. Oceanogr. 41, 2223 (2011).

[27] R. K. Walter, M. E. Squibb, C. B. Woodson, J. R. Koseff, and S. G. Monismith, Stratified turbulence in the nearshore coastal ocean: Dynamics and evolution in the presence of internal bores, J. Geophys. Res.: Oceans 119, 8709 (2014).

[28] W. G. Large, J. C. McWilliams, and S. C. Doney, Oceanic vertical mixing: A review and a model with a nonlocal boundary layer parameterization, Rev. Geophys. 32, 363 (1994).

[29] S. P. S. Arya, Buoyancy effects in a horizontal flat-plate boundary layer, J. Fluid Mech. 68, 321 (1975).

[30] R. E. Britter, An experiment on turbulence in a density stratified fluid, Ph.D. thesis, Monash University, Melbourne, Australia, 1974.

[31] J. F. Piat and E. J. Hopfinger, A boundary layer topped by a density interface, J. Fluid Mech. 113, 411 (1981).

[32] S. Komori, H. Ueda, F. Ogino, and T. Mizushina, Turbulence structure in stably stratified open-channel flow, J. Fluid Mech. 130, 13 (1983).

[33] K. Fukui, M. Nakajima, and H. Ueda, A laboratory experiment on momentum and heat transfer in the stratified surface layer, Q. J. R. Meteorol. Soc. 109, 661 (1983).

[34] R. P. Garg, J. H. Ferziger, S. G. Monismith, and J. R. Koseff, Stably stratified turbulent channel flows. I. Stratification regimes and turbulence suppression mechanism, Phys. Fluids 12, 2569 (2000).

[35] V. Armenio and S. Sarkar, An investigation of stably stratified turbulent channel flow using large-eddy simulation, J. Fluid Mech. 459, 1 (2002). 
[36] J. R. Taylor, S. Sarkar, and V. Armenio, Large eddy simulation of stably stratified open channel flow, Phys. Fluids 17, 116602 (2005).

[37] S. Basu and F. Porté-Agel, Large-eddy simulation of stably stratified atmospheric boundary layer turbulence: A scale-dependent dynamic modeling approach, J. Atm. Sci. 63, 2074 (2006).

[38] R. Stoll and F. Porté-Agel, Large-eddy simulation of the stable atmospheric boundary layer using dynamic models with different averaging schemes, Bound.-Layer Meteorol. 126, 1 (2008).

[39] X. Ruan, A. F. Thompson, and J. R. Taylor, The evolution and arrest of a turbulent stratified oceanic bottom boundary layer over a slope: Downslope regime, J. Phys. Oceanogr. 49, 469 (2019).

[40] O. Iida, N. Kasagi, and Y. Nagano, Direct numerical simulation of turbulent channel flow under stable density stratification, Int. J. Heat Mass Transf. 45, 1693 (2002).

[41] F. T. M. Nieuwstadt, Direct numerical simulation of stable channel flow at large stability, Bound.-Layer Meteorol. 116, 277 (2005).

[42] G. Brethouwer, P. Billant, E. Lindborg, and J.-M. Chomaz, Scaling analysis and simulation of strongly stratified turbulent flows, J. Fluid Mech. 585, 343 (2007).

[43] O. Flores and J. J. Riley, Analysis of turbulence collapse in the stably stratified surface layer using direct numerical simulation, Bound.-Layer Meteorol. 139, 241 (2011).

[44] M. García-Villalba and J. C. Del Alamo, Turbulence modification by stable stratification in channel flow, Phys. Fluids 23, 045104 (2011).

[45] T. Gerz, U. Schumann, and S. Elghobashi, Direct numerical simulation of stratified homogeneous turbulent shear flows, J. Fluid Mech. 200, 563 (1989).

[46] E. Itsweire, S. Holt, J. Koseff, and J. Ferziger, Direct numerical simulations of stably-stratified sheared turbulence: Implications for oceanic mixing, in Center for Turbulence Research Proceedings of the Summer Program (1990), pp. 163-180.

[47] S. E. Holt, J. R. Koseff, and J. H. Ferziger, A numerical study of the evolution and structure of homogeneous stably stratified sheared turbulence, J. Fluid Mech. 237, 499 (1992).

[48] J. Jiménez and P. Moin, The minimal flow unit in near-wall turbulence, J. Fluid Mech. 225, 213 (1991).

[49] Y. Hwang and C. Cossu, Self-sustained processes in the logarithmic layer of turbulent channel flows, Phys. Fluids 23, 061702 (2011).

[50] N. A. Bakas, P. J. Ioannou, and G. E. Kefaliakos, The emergence of coherent structures in stratified shear flow, J. Atm. Sci. 58, 2790 (2001).

[51] T. S. Eaves and C. P. Caulfield, Disruption of SSP/VWI states by a stable stratification, J. Fluid Mech. 784, 548 (2015).

[52] F. Gómez, H. M. Blackburn, M. Rudman, A. S. Sharma, and B. J. McKeon, A reduced-order model of three-dimensional unsteady flow in a cavity based on the resolvent operator, J. Fluid Mech. 798, R2 (2016).

[53] S. Symon, K. Rosenberg, S. T. M. Dawson, and B. J. McKeon, Non-normality and classification of amplification mechanisms in stability and resolvent analysis, Phys. Rev. Fluids 3, 053902 (2018).

[54] A. Towne, A. Lozano-Durán, and X. Yang, Resolvent-based estimation of space-time flow statistics, J. Fluid Mech. 883, A17 (2020).

[55] B. J. McKeon and A. S. Sharma, A critical-layer framework for turbulent pipe flow, J. Fluid Mech. 658, 336 (2010).

[56] Y. Hwang and C. Cossu, Self-Sustained Process at Large Scales in Turbulent Channel Flow, Phys. Rev. Lett. 105, 044505 (2010).

[57] C.-A. Yeh and K. Taira, Resolvent-analysis-based design of airfoil separation control, J. Fluid Mech. 867, 572 (2018).

[58] A. Towne, O. T. Schmidt, and T. Colonius, Spectral proper orthogonal decomposition and its relationship to dynamic mode decomposition and resolvent analysis, J. Fluid Mech. 847, 821 (2018).

[59] H. J. Bae, S. T. M. Dawson, and B. J. McKeon, Resolvent-based study of compressibility effects on supersonic turbulent boundary layers, J. Fluid Mech. 883, A29 (2020).

[60] R. M. McMullen, K. Rosenberg, and B. J. McKeon, Interaction of forced Orr-Sommerfeld and Squire modes in a low-order representation of turbulent channel flow, Phys. Rev. Fluids 5, 084607 (2020). 
[61] P. A. S. Nogueira, P. Morra, E. Martini, A. V. G. Cavalieri, and D. S. Henningson, Forcing statistics in resolvent analysis: Application in minimal turbulent Couette flow, J. Fluid Mech. 908, A32 (2021).

[62] B. J. McKeon, The engine behind (wall) turbulence: Perspectives on scale interactions, J. Fluid Mech. 817, P1 (2017).

[63] M. R. Jovanović, From bypass transition to flow control and data-driven turbulence modeling: An inputoutput viewpoint, Annu. Rev. Fluid Mech. 53, 311 (2021).

[64] P. Morra, P. A. Nogueira, A. V. Cavalieri, and D. S. Henningson, The colour of forcing statistics in resolvent analyses of turbulent channel flows, J. Fluid Mech. 907, A24 (2021).

[65] R. Moarref, A. S. Sharma, J. A. Tropp, and B. J. McKeon, Model-based scaling of the streamwise energy density in high-Reynolds-number turbulent channels, J. Fluid Mech. 734, 275 (2013).

[66] A. Madhusudanan, Coherent structures from the linearized Navier-Stokes equations for wall-bounded turbulent flows, Ph.D. thesis, University of Melbourne, 2020.

[67] E. N. Lorenz, Available potential energy and the maintenance of the general circulation, Tellus 7, 157 (1955).

[68] J. S. Turner, Buoyancy Effects in Fluids (Cambridge University Press, 1979).

[69] P. Orlandi, Fluid Flow Phenomena: A Numerical Toolkit (Springer, 2000).

[70] A. A. Wray, Minimal-storage time advancement schemes for spectral methods, Tech. Rep. ( NASA Ames Research Center, Mountain View, CA, 1990).

[71] A. J. Chorin, Numerical solution of the Navier-Stokes equations, Math. Comput. 22, 745 (1968).

[72] H. J. Bae, A. Lozano-Durán, S. T. Bose, and P. Moin, Dynamic slip wall model for large-eddy simulation, J. Fluid Mech. 859, 400 (2019).

[73] A. Lozano-Durán and H. J. Bae, Characteristic scales of Townsend's wall-attached eddies, J. Fluid Mech. 868, 698 (2019).

[74] L. N. Trefethen and M. Embree, Spectra and Pseudospectra (Princeton University Press, Princeton, NJ, 2005).

[75] J. H. Marques Ribeiro, C.-A. Yeh, and K. Taira, Randomized resolvent analysis, Phys. Rev. Fluids 5, $033902(2020)$.

[76] J. C. del Alamo, J. Jiménez, P. Zandonade, and R. D. Moser, Scaling of the energy spectra of turbulent channels, J. Fluid Mech. 500, 135 (2004).

[77] E. J. Hopfinger, Turbulence in stratified fluids: A review, J. Geophys. Res. 92, 5287 (1987).

[78] J. L. Lumley, The structure of inhomogeneous turbulent flows, in Atmospheric Turbulence and Radio Wave Propagation, edited by A. M. Yaglom and V. I. Tatarsky (Nauka, Moscow, 1967).

[79] S. J. Kline, W. C. Reynolds, F. A. Schraub, and P. W. Runstadler, The structure of turbulent boundary layers, J. Fluid Mech. 30, 741 (1967).

[80] C. R. Smith and S. P. Metzler, The characteristics of low-speed streaks in the near-wall region of a turbulent boundary layer, J. Fluid Mech. 129, 27 (1983).

[81] D. J. Tritton, Some new correlation measurements in a turbulent boundary layer, J. Fluid Mech. 28, 439 (1967).

[82] Z. Liu, R. J. Adrian, and T. J. Hanratty, Large-scale modes of turbulent channel flow: Transport and structure, J. Fluid Mech. 448, 53 (2001).

[83] B. Ganapathisubramani, N. Hutchins, W. T. Hambleton, E. K. Longmire, and I. Marusic, Investigation of large-scale coherence in a turbulent boundary layer using two-point correlations, J. Fluid Mech. 524, 57 (2005).

[84] J. H. Lee and H. J. Sung, Very-large-scale motions in a turbulent boundary layer, J. Fluid Mech. 673, 80 (2011).

[85] S. Pirozzoli and M. Bernardini, Turbulence in supersonic boundary layers at moderate Reynolds number, J. Fluid Mech. 688, 120 (2011).

[86] J. A. Sillero, J. Jiménez, and R. D. Moser, Two-point statistics for turbulent boundary layers and channels at Reynolds numbers up to $\delta^{+} \approx 2000$, Phys. Fluids 26, 105109 (2014).

[87] S. Symon, S. J. Illingworth, and I. Marusic, Energy transfer in turbulent channel flows and implications for resolvent modelling, J. Fluid Mech., 911, A3 (2021). 
[88] Y. Mizuno, Spectra of energy transport in turbulent channel flows for moderate Reynolds numbers, J. Fluid Mech. 805, 171 (2016).

[89] R. Moarref, M. R. Jovanović, J. A. Tropp, A. S. Sharma, and B. J. McKeon, A low-order decomposition of turbulent channel flow via resolvent analysis and convex optimization, Phys. Fluids 26, 051701 (2014).

[90] K. Rosenberg and B. J. McKeon, Efficient representation of exact coherent states of the Navier-Stokes equations using resolvent analysis, Fluid Dyn. Res. 51, 011401 (2019). 